\title{
Axion-CMB scenario in a supercooled universe
}

\author{
Satoshi Iso, ${ }^{1,2, *}$ Kiyoharu Kawana, ${ }^{1,3, \dagger}$ and Kengo Shimada ${ }^{1, *}$ \\ ${ }^{1}$ Theory Center, High Energy Accelerator Research Organization (KEK), \\ Tsukuba, Ibaraki 305-0801, Japan \\ ${ }^{2}$ Graduate University for Advanced Studies (SOKENDAI), Tsukuba, Ibaraki 305-0801, Japan \\ ${ }^{3}$ Center for Theoretical Physics, Department of Physics and Astronomy, Seoul National University, \\ Seoul 08826, Korea
}

(Received 16 May 2021; accepted 15 August 2021; published 14 September 2021)

\begin{abstract}
"Axion-CMB scenario" is an interesting possibility to explain the temperature anisotropy of the cosmic microwave background (CMB) by primordial fluctuations of the QCD axion [S. Iso, K. Kawana, and K. Shimada, Phys. Rev. D 102, 103513 (2020)]. In this scenario, fluctuations of radiations are generated by an energy exchange between axions and radiations, which results in the correlation between the primordial axion fluctuations and the CMB anisotropies. Consequently, the cosmological observations stringently constrain a model of the axion and the early history of the universe. In particular, we need a large energy fraction $\Omega_{A}$ of the axion at the QCD phase transition, but it must become tiny at the present universe to suppress the isocurvature power spectrum. One of natural cosmological scenarios to realize such a situation is the thermal inflation which can sufficiently dilute the axion abundance. Thermal inflation occurs in various models. In this paper, we focus on a classically conformal (CC) $B-L$ model with a QCD axion. In this model, the early universe undergoes a long supercooling era of the $B-L$ and electroweak symmetries, and thermal inflation naturally occurs. Thus it can be a good candidate for the axion-CMB scenario. But the axion abundance at the QCD transition is shown to be insufficient in the original CC $B-L$ model. To overcome the situation, we extend the model by introducing $N$ scalar fields $S$ (either massive or massless) and consider a novel cosmological history such that the $O(N)$ and the $B-L$ sectors evolve almost separately in the early universe. We find that all the necessary conditions for the axion-CMB scenario can be satisfied in some parameter regions for massless $S$ fields, typically $N \sim 10^{19}$ and the mass of $B-L$ gauge boson around $5-10 \mathrm{TeV}$.
\end{abstract}

DOI: 10.1103/PhysRevD.104.063525

\section{INTRODUCTION}

Anisotropy of the cosmic microwave background $(\mathrm{CMB})$ is one of the most fascinating subject in the particle cosmology since it is generated at an early stage of the history of the Universe and possibly related to the physics beyond the Standard Model (BSM). The current observational data such as Planck 2018 [1-3] tells us that the temperature fluctuation is almost scale invariant and adiabatic, which favors inflation models by a single scalar field. But it is is not the unique scenario for explaining the CMB anisotropy. A well-known example is the curvaton scenario [4-14] where the origin of fluctuations comes

\footnotetext{
iso@post.kek.jp

kawana@snu.ac.kr

kengo.shimada@kek.jp
}

Published by the American Physical Society under the terms of the Creative Commons Attribution 4.0 International license. Further distribution of this work must maintain attribution to the author(s) and the published article's title, journal citation, and DOI. Funded by SCOAP ${ }^{3}$. from an additional scalar field called curvaton. In this scenario, the primordial fluctuation of curvaton is converted to that of radiation through decay of curvatons. An interesting aspect of the curvaton model is a prediction of sizable non-Gaussianities.

Axion-CMB scenario is similar to the curvaton scenario where $\mathrm{CMB}$ anisotropy is induced by the primordial perturbations of the QCD axion [15]. In this scenario, Peccei-Quinn symmetry [16-24] is assumed to be already broken before the primordial inflation, and the axion field acquires primordial fluctuations during the inflation. As the universe cools down to the QCD scale $T_{\mathrm{QCD}}$, the axion potential is generated by transferring non-zero energy from radiation. Then the primordial axion fluctuations are converted to the density fluctuation of the radiation. For a successful realization of the scenario, three conditions must be satisfied: (1) A large amount of energy density of axions at $T=T_{\mathrm{QCD}}$ is necessary to suppress nonGaussianity. (2) Axions must be largely diluted until present in order to satisfy the isocurvature constraint. (3) In addition, it must explain the observed CMB 
amplitude. These three conditions require something like thermal inflation [25-28] after the QCD temperature [15]. Indeed, under a couple of reasonable assumptions, we found that they can be satisfied as long as the thermal inflation lasts long enough after the QCD phase transition.

The purpose of this paper is to present a concrete particle physics model that can realize such a thermal inflation. In this paper, we will consider the classically conformal $B-L$ model [29-32] with a QCD axion and its extension with $O(N)$ scalar fields $S$. Originally, the $B-L$ model with classical conformality was proposed to explain the eletroweak (EW) scale by Coleman-Weinberg (CW) mechanism [29-31,33,34]. Besides, it was pointed out that this model predicts a long supercooling era of the $B-L$ and EW symmetries due to the classical conformality of the scalar potential [32]. The supercooling lasts below $T_{\mathrm{QCD}}$ and the energy density of radiation becomes smaller than the vacuum energy of the false vacuum. Thus thermal inflation occurs even after the QCD phase transition and axions can be sufficiently diluted after the QCD phase transition. But there is one technical difficulty to obtain a large value of the axion abundance at $T=T_{\mathrm{QCD}}$. It is because the Higgs vacuum expectation value (vev), $\left\langle\left. h\right|_{T_{\mathrm{QCD}}} \sim \Lambda_{\mathrm{QCD}}\right.$, which is responsible for the nonzero axion potential, is generated by quark condensates $\langle\bar{q} q\rangle$ and becomes of order $100 \mathrm{MeV}$. As we will see, it is too small for the necessary value of the axion abundance. In order to overcome this difficulty, we need an additional mechanism to raise $\left.\langle h\rangle\right|_{T_{\mathrm{QCD}}}$ to at least $100 \mathrm{GeV}$.

For this purpose, we introduce $O(N)$ scalar fields $S$ coupled to the Higgs field with a very weak negative coupling, and consider a novel history of the early universe: we suppose that the $O(N)$ sector has evolved almost separately from our universe, with a much higher temperature $\tilde{T}$ than that of our universe. Then the negative coupling will generate sizable negative thermal corrections to the Higgs quadratic potential [35-42], and $\left.\langle h\rangle\right|_{T_{\mathrm{QCD}}}$ can become as large as $100 \mathrm{GeV}$. In the paper, we will consider two extremal cases, very massive $S$ with $m_{S} \gg m_{Z^{\prime}}$ or massless $S$. We show that various observational constraints, especially sufficient dilution of axions and $S$ particles, can be simultaneously satisfied in the massless case for $N \gtrsim 10^{19}$, but not in the massive case. One might think that such a large number of degrees of freedom (dof) would be inconsistent with various phenomenological aspects such as collider observables. This is actually problematic as long as we focus on moderate values of the portal coupling $\lambda_{S H}$. However, as discussed in Refs. [36,38], it is possible to make a model consistent by taking $\tilde{\lambda}_{S H}:=N \lambda_{S H}$ as a free parameter and choosing moderate values of $\tilde{\lambda}_{S H}$. Roughly speaking, collider observables are determined by the combination $N \lambda_{S H}^{2}$ and this is suppressed by $1 / N$ when we fix $\tilde{\lambda}_{S H}$. See Refs. [36,38] for more detailed discussion.
The organization of the paper is as follows. In Sec. II, we first summarize the setup and a typical set of parameters of our model. In Sec. III, we review the axion-CMB scenario [15] and explain what conditions are necessary for the scenario to be observationally viable. In Sec. IV, we focus on a specific particle physics model of the axion-CMB scenario, a classically conformal (CC) $B-L$ model since the model predicts thermal inflation below $T_{\mathrm{QCD}}$. We first see that the original CC $B-L$ model has a problem as a candidate for the axion-CMB scenario. We then propose an extended model to overcome this difficulty by introducing an additional $O(N)$ scalar field $S$, and investigate the model for either large $m_{S} \gg m_{Z^{\prime}}$ or $m_{S}=0$. Our analysis shows that the massless case can satisfy all the necessary conditions for the model to be phenomenologically viable.

\section{SCHEMATIC PICTURE}

Before going into details, we summarize the setup of our particle physics model and its cosmological history of the early universe. We then provide an example set of parameters of the model that can realize a phenomenologically viable axion-CMB scenario. Our particle physics model consists of, besides standard model (SM) particles,

(i) axion field $A$

(ii) $B-L U(1)$ gauge field $Z^{\prime}$ with gauge coupling $g_{B-L}$, right-handed neutrinos to cancel $U(1)_{B-L}$ gauge anomaly, and SM singlet scalar $\phi$ with $U(1)_{B-L}$ charge.

(iii) $O(N)$ scalars $S$ with very weak scalar mixings of $\lambda_{S H}=-\left|\lambda_{S H}\right|$ and $\lambda_{S \phi}$.

Our basic assumption of the model is classical conformality: there are no quadratic terms in the scalar potential so that the EW phase transition has experienced a long supercooling era and is triggered by QCD chiral symmetry breaking $(\chi \mathrm{SB}) . O(N)$ scalars are introduced to enhance the vev of Higgs field by the inverse symmetry breaking (ISB) mechanism [35] owing to the negative scalar mixing $\lambda_{S H}$. A typical set of parameters that can satisfy various observational constraints is given, for example, by

$$
g_{B-L} \sim 0.1, \quad m_{Z^{\prime}} \sim 10 \mathrm{TeV}, \quad N \sim 10^{19}, \quad\left|\lambda_{S H}\right| N \sim 1 .
$$

Though $N$ is huge, it is phenomenologically allowed due to the very weak mixing with the SM. This model exhibits a peculiar thermal history of the early universe;

(i) The universe has experienced a long supercooling era and the EW phase transition is triggered by $\mathrm{QCD} \chi \mathrm{SB}$.

(ii) Thermal inflation occurs when the temperature of the $O(N)$ sector $\tilde{T}$ is around $m_{Z^{\prime}} / N^{1 / 4}$. After the thermal inflation ends, the universe is reheated and then the ordinary big bang universe begins.

(iii) $\tilde{T}$ has evolved separately from the $\mathrm{SM}$ and $B-L$ sector due to the very weak mixings. 


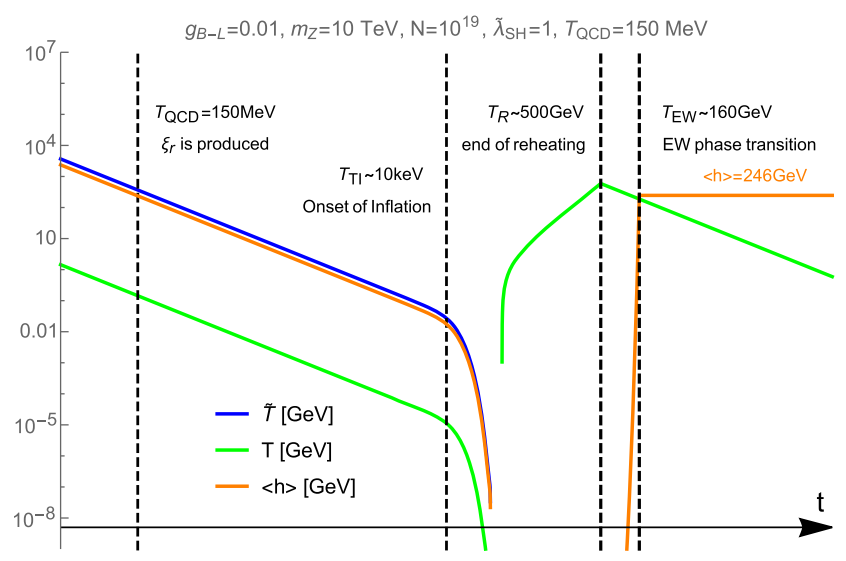

FIG. 1. Evolutions of the temperatures of the two separate sectors. The blue (green) corresponds to the $O(N)$ (SM) sector. Here we also show the evolution of the Higgs vev by orange.

In Fig. 1, we plot evolutions of temperatures of the two separate sectors. The green (blue) lines represent temperatures of the SM (and $B-L$ ) sector $T$, and $O(N)$ sector $\tilde{T}$, respectively. Before thermal inflation, $O(N)$ sector has higher temperature than the SM sector so that thermal effect of $\tilde{T}$ with a negative coupling gives the Higgs field a large nonvanishing vev. In the figure, we also plot the vev of Higgs $\langle h\rangle$, which is enhanced to $100 \mathrm{GeV}$ when the curvature perturbation $\zeta_{r}$ is produced via the transfer from the axion field fluctuation. After thermal inflation, no $O(N)$ sector particles are produced and $\tilde{T} \sim 0$. In the big bang universe after thermal inflation, the EW phase transition takes place again in the standard manner with the Higgs negative mass term due to the vev of the singlet $\phi$. The thermal inflation dilutes the axions and suppresses the isocurvature fluctuations at the present universe. Sufficient amount of CMB fluctuations can be produced due to the enhancement of Higgs vev $\langle h\rangle$ by the ISB mechanism, since larger vev gives a larger axion potential.

\section{AXION-CMB SCENARIO}

In this section, we summarize the axion-CMB scenario [15]. It is similar to the curvaton scenario, but the transfer mechanisms of the fluctuations from the curvaton (or axion) to radiation are different. Curvatons decay into radiation to generate the $\mathrm{CMB}$ anisotropy while axions are assumed to be stable until present. In the axion-CMB scenario, the fluctuations of radiation are induced when the axion potential is generated at the QCD phase transition. Such scenario is discussed in Ref. [43] but it is shown to be inconsistent with observations as far as the standard cosmology is concerned. The reason is the following. For the scenario to be observationally viable, the model must satisfy the following three conditions:

(1) production of sufficient amplitudes of the CMB anisotoropy

(2) consistency with the axion isocurvature constraint
(3) consistency with the non-Gaussianity constraint. The conditions, 1 and 3, require that a sufficiently large energy fraction $\Omega_{A}$ of the axion is present at the QCD scale when the axion-potential is generated. On the other hand, the axion abundance must be tiny at the present era to satisfy the isocurvature constraint. In order to satisfy them simultaneously, we need a mechanism to dilute axions after the QCD phase transition such as low scale thermal inflation.

The axion-CMB scenario is effectively parametrized by three parameters:

(i) Amplitude of the primordial fluctuations of axion $(\delta A / \bar{A})^{2} \sim H_{\text {exit }}^{2} /\left(f_{A} \theta\right)^{2}$,

(ii) ratio of energy densities of axion to that of radiation right after the axion potential is generated, $R=\Omega_{A} / \Omega_{r}$,

(iii) fraction of the axion abundance in the total cold dark matter (CDM) today, $r_{A}=\Omega_{A} /\left.\Omega_{\mathrm{CDM}}\right|_{\text {today }}$.

The three conditions constrain the allowed region of the three three parameters, together with the initial misalignment angle $\theta_{\text {ini }}$. We first summarize them in the following. See Ref. [15] for more details.

(i) $\mathrm{CMB}$ amplitude QCD-like axion $A$ is assumed to be massless during the primordial inflation and fluctuates with the amplitude $\delta A_{\text {ini }}$;

$$
\begin{aligned}
\left\langle\delta A_{\text {ini }}(k) \delta A_{\text {ini }}\left(k^{\prime}\right)\right\rangle & =(2 \pi)^{3} \delta^{(3)}\left(k+k^{\prime}\right) \frac{H_{\text {exit }}^{2}(k)}{2 k^{3}}, \\
H_{\text {exit }}(k) & :=\left.H\right|_{k=a \bar{H}} .
\end{aligned}
$$

The axion acquires potential $V_{A}(A)$ at the QCD temperature and becomes massive. In the generation of the axion potential, increase of the potential energy is compensated by decrease of radiation energy. Thus the primordial axion fluctuations induce the density fluctuations of radiations. Suppose that the dominant part of the density fluctuations of radiation originate in this induced fluctuations and their initial curvature perturbation is negligible. Then the curvature perturbation is given by the axion fluctuation as

$$
\zeta_{r} \sim \frac{R}{4} \frac{\delta V_{A}}{V_{\bar{A}}} \delta A_{\text {ini }}
$$

where $V_{\bar{A}}=V_{A}(A=\bar{A})$ and

$$
R:=\left.\frac{\rho_{A}}{\rho_{r}}\right|_{T_{\mathrm{QCD}}}
$$

is the ratio of the energy densities of the axion to radiation evaluated right after the potential is instantaneously generated at $T=T_{\mathrm{QCD}}$. If $\rho_{A}$ is dominated by the potential energy of QCD axion 
at this moment, $R$ can be calculated as [15]

$$
\begin{aligned}
& R=\left.\frac{V_{A}(\bar{A})}{\rho_{r}}\right|_{T=T_{\mathrm{QCD}}} \\
& \simeq \frac{30}{\pi^{2} g_{\mathrm{QCD}}} \frac{m_{u} / m_{d}}{\left(1+m_{u} / m_{d}\right)^{2}} \frac{m_{\pi}^{2} f_{\pi}^{2}}{T_{\mathrm{QCD}}^{4}}\left(1-\cos \left(\bar{\theta}_{\mathrm{ini}}\right)\right) \\
& \simeq 0.012 \times\left(\frac{150 \mathrm{MeV}}{T_{\mathrm{QCD}}}\right)^{4}\left(\frac{\langle h\rangle}{246 \mathrm{GeV}}\right)\left(1-\cos \left(\bar{\theta}_{\mathrm{ini}}\right)\right),
\end{aligned}
$$

where $g_{\mathrm{QCD}}=69 / 4$ is the effective number of dof right after the QCD phase transition to which pions also contribute. Note that the Higgs vev is treated as a free parameter in the above equation since this point becomes the most crucial in discussing an explicit realization of the scenario in a concrete particle physics model discussed in the next section.

The above curvature perturbation $\zeta_{r}$ is passed down to the current density of radiation if there are no further mixings with other fields, and the CMB amplitude is given by

$$
\sqrt{A_{s}}=R \frac{H_{\mathrm{exit}}\left(k_{*}\right)}{4 \pi f_{A} \bar{\theta}_{\mathrm{ini}}}=\sqrt{2.1 \times 10^{-9}}=4.6 \times 10^{-5},
$$

where $k_{*}=0.05 \mathrm{Mpc}^{-1}$ is the reference (pivot) scale. This gives a relation between $R$ and $H_{\text {exit }}\left(k_{*}\right) / \pi f_{A}$ for each misalignment angle $\bar{\theta}_{\text {ini }}$.

(ii) Non-Gaussianity The anharmonicity of the axion potential produces non-Gaussianities of the $\mathrm{CMB}$ anisotropy. Following Ref. [15], we have

$$
f_{\mathrm{NL}} \sim-\frac{10}{3 R} \frac{\cos \left(\bar{\theta}_{\text {ini }}\right)\left(1-\cos \left(\bar{\theta}_{\text {ini }}\right)\right)}{\sin ^{2}\left(\bar{\theta}_{\text {ini }}\right)}-\frac{10}{3},
$$

$g_{\mathrm{NL}} \sim-\frac{1}{6}\left(\frac{20}{3 R}\right)^{2} \tan ^{2}\left(\frac{\bar{\theta}_{\mathrm{ini}}}{2}\right)+\frac{20}{3} f_{\mathrm{NL}}-\frac{1}{6}\left(\frac{20}{3}\right)^{2}$.

The non-Gaussianities are inversely proportional to $R$, because both of the leading Gaussian fluctuation and subleading nonlinear parts are proportional to $R(<1)$. Consequently non-Gaussiannities, by definition, become tiny for a small value of $R$. Thus the observational non-Gaussianity constraints favor large $R$ region.

In Fig. 2, we plot the allowed region of $\left(\bar{\theta}_{\text {ini }}, R\right)$ determined by the observational bounds for the nonGaussianity [3]

$$
f_{\mathrm{NL}}^{\text {local }}=4 \pm 20, \quad g_{\mathrm{NL}}=(-5.8 \pm 13) \times 10^{4},
$$

(95\% CL by Planck 2018).

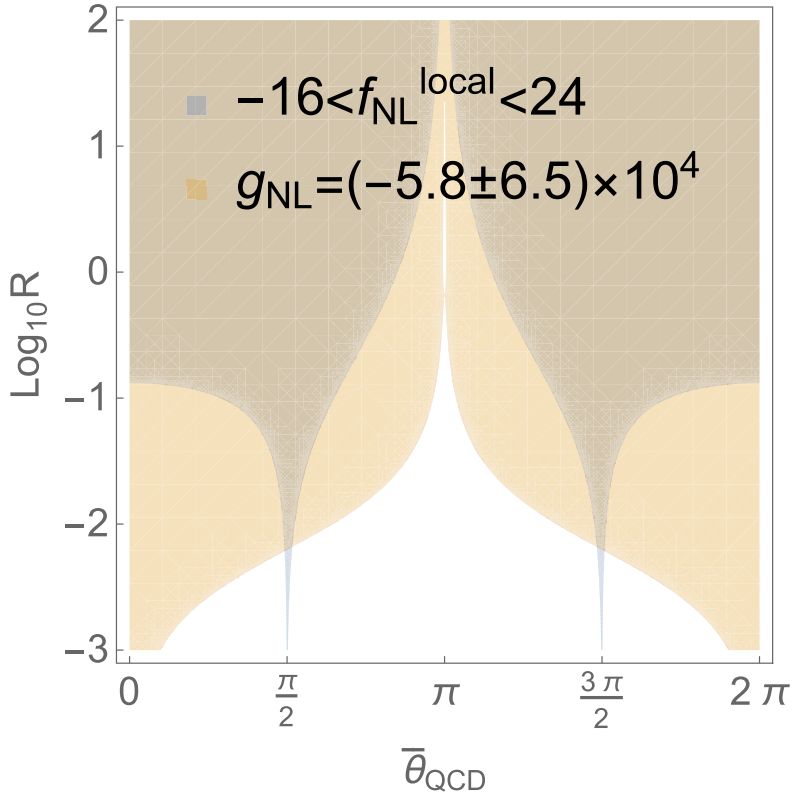

FIG. 2. Allowed regions of $\left(\bar{\theta}_{\mathrm{QCD}}, R\right)$ by the non-Gaussianity constraints, Eq. (9). The blue (orange) region represents the region allowed by $f_{\mathrm{NL}}\left(g_{\mathrm{NL}}\right) \cdot \bar{\theta} \sim \pi / 2,3 \pi / 2$ is necessary to obtain $R \sim \mathcal{O}(0.01)$, where $V^{\prime \prime}$ vanishes.

For more details, see Ref. [15]. The blue (orange) region corresponds to $f_{\mathrm{NL}}\left(g_{\mathrm{NL}}\right)$. One can see that, as long as $\bar{\theta}_{\text {ini }} \sim 0$, the lower bound of $R$ is $\mathcal{O}(0.1)$, but the bound can be reduced to $\mathcal{O}(0.01)$ around $\bar{\theta}_{\text {ini }}=$ $\pi / 2$ or $3 \pi / 2$. From Eq. (5), these lower bounds of $R$ requires the Higgs vev $\langle h\rangle$ at the QCD phase transition to be larger than $\mathcal{O}(1) \mathrm{TeV}$ for $\theta_{\text {ini }} \sim 0$, or larger than $\mathcal{O}\left(10^{2}\right) \mathrm{GeV}$ for $\theta_{\mathrm{ini}} \sim \pi / 2$ or $3 \pi / 2$. Using Eq. (6), the bound $R \gtrsim 0.01$ corresponds to

$$
\frac{H_{\text {exit }}\left(k_{*}\right)}{4 \pi f_{A} \bar{\theta}_{\text {ini }}}<4.6 \times 10^{-3} .
$$

(iii) Isocurvature perturbations The fluctuation of the axion field also produces isocurvature perturbations as in the standard cosmology of QCD-axion [43-50]. The isocurvature power spectrum is calculated as

$$
\mathcal{P}_{\mathcal{I I}}(k)=\frac{k^{3}}{2 \pi^{2}} P_{\mathcal{I}}(k)=\left(\frac{r_{A} H_{\text {exit }}(k)}{\pi f_{A} \bar{\theta}_{\text {ini }}}\right)^{2},
$$

where $r_{A}$ is the ratio of the abundance of the axion to the total cold dark matter (CDM) today,

$$
r_{A}:=\left.\frac{\Omega_{A}}{\Omega_{\mathrm{CDM}}}\right|_{\text {today }},
$$

where $\Omega_{\mathrm{CDM}}$ denotes the energy fraction of CDM. By plugging this into the isocurvature constraint [2], 
$\beta_{\text {iso }}(k)=\frac{\mathcal{P}_{\mathcal{I I}}}{\mathcal{P}_{\mathcal{R R}}+\mathcal{P}_{\mathcal{I I}}}<0.00107$ for $\cos \Delta=-1$,

$\cos \Delta=\frac{\mathcal{P}_{\mathcal{R I}}}{\sqrt{\mathcal{P}_{\mathcal{R R}} \mathcal{P}_{\mathcal{I I}}}}$,

and using $\mathcal{P}_{\mathcal{R} \mathcal{R}}\left(k_{*}\right)=2.1 \times 10^{-9}$, we obtain the following constraint

$\frac{r_{A} H_{\text {exit }}\left(k_{*}\right)}{\pi f_{A} \bar{\theta}_{\text {ini }}}<1.5 \times 10^{-6}$ for $\cos \Delta=-1$.

In particular, by eliminating $H_{\text {exit }}\left(k_{*}\right) /\left(f_{A} \bar{\theta}_{\text {ini }}\right)$ from Eqs. (6) and (14), we obtain an inequality between $r_{A}$ and $R$ as

$$
r_{A}<8.2 \times 10^{-3} R
$$

which shows that the axion abundance has to be sufficiently diluted after the QCD transition. Thus we need a mechanism such as thermal inflation at the QCD scale.

In the previous paper [15], we have studied thermal inflation scenario and shown that all the necessary conditions can be satisfied under reasonable assumptions. Thus, a next step is to construct a concrete model of particle physics. In the next section, we will consider the classically conformal $B-L$ model [2932] with a QCD axion because the model undergoes supercooling of the $B-L$ and EW symmetries, and low scale thermal inflation naturally occurs. However, as we will see in the next section, the model predicts a small Higgs vev $\left.\langle h\rangle\right|_{T_{\mathrm{QCD}}} \sim \Lambda_{\mathrm{QCD}}$ when the axion potential is generated. Consequently the height of the axion potential is too low to get a sufficiently large value of $R \sim 0.01$, and the model is already excluded from the non-Gaussianity constraint. Hence, we need some modifications of the original $B-L$ model so that $\left.\langle h\rangle\right|_{T_{\mathrm{OCD}}}$ becomes at least $\mathcal{O}\left(10^{2}\right) \mathrm{GeV}$, or larger.

\section{PARTICLE PHYSICS MODELS OF AXION-CMB SCENARIO}

As we saw in the previous section, we need a larger value of $R$ than $\sim 0.01$, which corresponds to $\left.\langle h\rangle\right|_{T_{\mathrm{QCD}}} \sim$ $\mathcal{O}\left(10^{2}\right) \mathrm{GeV}$. In addition, a dilution mechanism like thermal inflation is necessary to satisfy a small value of $r_{A} ; r_{A}<8.2 \times 10^{-3} R$. In this section, we consider a classically conformal $B-L$ model since the low scale thermal inflation naturally occurs.

\section{A. Classically conformal $B-L$ model}

The classically conformal (CC) $B-L$ model [29-32] is an extension of the SM with the right handed neutrinos $N_{i}$, the $B-L$ gauge boson $Z^{\prime}$, and the $B-L$ scalar $\Phi$ which breaks the $B-L$ gauge symmetry by Coleman-Weinberg
(CW) mechanism. Real components of scalar fields are represented as $h$ and $\phi$ respectively. We first summarize the thermal history of the early universe of the model.

For this purpose we need the behavior of the scalar potential. At zero-temperature, the effective potential is given, at one-loop level, by [32]

$V=V_{\mathrm{TI}}+\lambda_{H}\left(H^{\dagger} H\right)^{2}-\lambda_{\phi H}\left(H^{\dagger} H\right)\left(\Phi^{\dagger} \Phi\right)+V_{\mathrm{CW}}(\Phi, H)$.

The SM and $B-L$ sectors couple through the $U(1)$ gauge and the scalar mixings. We will focus on the $\mathrm{CW}$ potential for the $\phi$ field by setting $h=0$,

$$
\begin{aligned}
V_{\mathrm{CW}}(\phi) & =\frac{B}{32 \pi^{2}} \phi^{4} \ln \left(\frac{\phi}{v_{\phi} e^{1 / 4}}\right), \\
B & \simeq 3\left(2 g_{B-L}\right)^{4}-2 \operatorname{Tr}\left[\left(\hat{Y}_{N} / \sqrt{2}\right)^{4}\right] .
\end{aligned}
$$

Here, $g_{B-L}$ is the $B-L$ gauge coupling, $Y_{N, i j}$ is the Yukawa coupling between $\Phi$ and $N_{i}$, and $v_{\phi}$ represents the minimum of the potential $V_{\mathrm{CW}}(\phi)$. The vev will be shifted by the scalar mixing in a nonzero Higgs vev, but its effect to $V(\phi)$ is tiny for $\langle h\rangle \ll\langle\phi\rangle$ and neglected in the following discussions. The parameter $V_{\mathrm{TI}}$ is chosen so that the total vacuum energy vanishes:

$$
V_{\mathrm{TI}}=\frac{B}{128 \pi^{2}} v_{\phi}^{4},
$$

which determines the Hubble scale of thermal inflation discussed below. The $B-L$ symmetry breaking triggers the EW symmetry breaking via the scalar mixing with a negative coefficient. Since the Higgs potential becomes

$$
\frac{\lambda_{H}}{4}\left(h^{4}-\frac{\lambda_{\phi H}}{\lambda_{H}} v_{\phi}^{2} h^{2}\right)=\frac{\lambda_{H}}{4}\left(h^{2}-\frac{\lambda_{\phi H}}{2 \lambda_{H}} v_{\phi}^{2}\right)^{2}-\frac{\lambda_{\phi H}^{2}}{4 \lambda_{H}} v_{\phi}^{4},
$$

the Higgs vev is given by

$$
v_{h}=\sqrt{\frac{\lambda_{\phi H}}{2 \lambda_{H}}} \times v_{\phi}=246 \mathrm{GeV},
$$

which gives a relation between $\lambda_{\phi H}$ and $v_{\phi}$.

Next, let us consider its thermal effects. Including the thermal correction of $Z^{\prime}$ and $N_{i}$, the high temperature expansion of one-loop effective potential of $\phi$ is given by [32]

$$
V_{1 \text { loop }}(\phi)=\frac{c_{2}}{2} T^{2} \phi^{2}-\frac{c_{3}}{3} T \phi^{3}+\frac{\tilde{B}}{4} \phi^{4},
$$

where 


$$
\begin{aligned}
c_{2}= & g_{B-L}^{2}+\frac{1}{24} \operatorname{Tr}\left(\hat{Y}_{N}^{\dagger} \hat{Y}_{N}\right), \quad c_{3}=\frac{6}{\pi} g_{B-L}^{3}, \\
\tilde{B}= & \frac{1}{8 \pi^{2}}\left\{3\left(2 g_{B-L}\right)^{4} \ln \left(\frac{T}{m_{Z^{\prime}}}\right)\right. \\
& \left.-2 \operatorname{Tr}\left[(\hat{Y} / \sqrt{2})^{4} \ln \left(\frac{T}{m_{N} c}\right)\right]\right\},
\end{aligned}
$$

and

$$
\begin{aligned}
m_{Z^{\prime}} & =2 g_{B-L} v_{\phi}, \quad m_{N}=Y_{N} v_{\phi} / \sqrt{2}, \\
c & =\left(e \alpha_{B}\right)^{-1 / 2}, \quad \log \alpha_{B}^{-1 / 2}=-\log 4 \pi+\gamma_{E} .
\end{aligned}
$$

In the following, we assume $g_{B-L} \gg Y_{N}$ so that we can neglect the contributions from $N_{i}$ for simplicity. At high temperature, the potential has a minimum at $\phi=h=0$. When $T$ decreases, a new minimum appears and, below $T_{c}=c e^{4 / 3} m_{Z^{\prime}}$, the potential height at the new minimum becomes lower. In usual cases, the false minimum at $\phi=0$ becomes unstable around this temperature. But in the class of models with classical conformality, there are no quadratic terms in the zero-temperature scalar potential, and the coefficient of the quadratic term of finite-temperature potential is always positive, and hence the false vacuum $\phi=0$ remains the local minimum at any smaller temperature below $T_{c}$; the early universe experiences a long supercooling era of the would-be broken symmetries. Finally quantum tunneling or some other effects destabilize the false vacuum.

During the supercooling era, temperature of the universe drops preserving $B-L$ and EW symmetries, and when the vacuum energy in Eq. (18) dominates the radiation energy, thermal inflation begins. By using Eqs. (17) and (18), the temperature when the thermal inflation starts is given by

$$
T_{\mathrm{TI}}=\left(\frac{30 V_{\mathrm{TI}}}{\pi^{2} g_{\mathrm{TI}}}\right)^{1 / 4}=\left(\frac{45}{64 g_{\mathrm{TI}}}\right)^{1 / 4} \frac{m_{Z^{\prime}}}{\pi},
$$

where $g_{\mathrm{TI}}$ is the degrees of freedom (dof) at $T=T_{\mathrm{TI}}$. As the Hubble scale during the thermal inflation, we have

$$
H_{\mathrm{TI}}=\frac{V_{\mathrm{TI}}^{1 / 2}}{\sqrt{3} M_{p l}} .
$$

At the QCD critical temperature $T_{\mathrm{QCD}} \sim \Lambda_{\mathrm{QCD}} \sim$ $150 \mathrm{MeV}$, chiral condensation occurs and the Higgs potential acquires an additional linear term via the top Yukawa coupling [51]

$$
-\frac{y_{t}}{\sqrt{2}}\langle\bar{t} t\rangle h \sim-\frac{y_{t}}{\sqrt{2}} \Lambda_{\mathrm{QCD}}^{3} h .
$$

Then the minimum of the Higgs field potential is shifted to have a nonzero vev,

$$
\langle h\rangle=v_{\mathrm{QCD}}:=\left(y_{t}\langle\bar{t} t\rangle / \sqrt{2}\right)^{1 / 3} \sim \Lambda_{\mathrm{QCD}} .
$$

Simultaneously, axion field acquires a potential through QCD nonperturbative effects. We now figure out why it is difficult to realize the axion-CMB scenario in the original $B-L$ model. As we discussed in the previous section, the lower bound of $R$ is $\mathcal{O}(0.01)$ due to the nonGaussianity constraints, and the Higgs vev in Eq. (5) must be $\mathcal{O}(100)$ Gev. Hence the above vev $\langle h\rangle \sim \Lambda_{\mathrm{QCD}}$ is too small to be consistent with the non-Gaussianity constraint. In the next subsection, we will extend the model by introducing additional $O(N)$ scalar in order to evade this difficulty.

Even after the Higgs acquires the QCD scale vev at $T=T_{\mathrm{QCD}}$, the thermal inflation continues because $\phi$ field remains at $\phi=0$, if $m_{Z^{\prime}} \geq \sqrt{2} m_{H}$. It can be seen by looking at the quadratic term of the finite temperature potential of $\phi$ in presence of $\langle h\rangle=v_{\mathrm{QCD}}$;

$\frac{T^{2} M_{Z^{\prime}}(\phi)^{2}}{8}-\frac{\lambda_{\phi H}}{4} v_{\mathrm{QCD}}^{2} \phi^{2}=\frac{m_{Z^{\prime}}^{2}}{8 v_{\phi}^{2}}\left(T^{2}-2\left(\frac{m_{H}}{m_{Z^{\prime}}}\right)^{2} v_{\mathrm{QCD}}^{2}\right) \phi^{2}$,

where $m_{H}=125 \mathrm{GeV}$ is the Higgs mass. If $m_{Z^{\prime}} \geq \sqrt{2} m_{H}$, the coefficient is positive at $T=T_{\mathrm{QCD}}$, and the scalar field is trapped at the false vacuum until the coefficient of quadratic term of $\phi$ becomes negative. The temperature at which the coefficient becomes negative and the thermal inflation ends ${ }^{1}$ is given by

$$
T_{\text {end }}=\frac{m_{H}}{\sqrt{2} m_{Z^{\prime}}} v_{\mathrm{QCD}}
$$

The $e$-folding number after $T=T_{\mathrm{QCD}}$ is now given by

$$
\Delta N_{\mathrm{QCD}}=\ln \left(\frac{T_{\mathrm{QCD}}}{T_{\text {end }}}\right)=\ln \left(\frac{\sqrt{2} m_{Z^{\prime}}}{m_{H}}\right) .
$$

\section{B. $O(N)$ scalar extension of the $\mathrm{CC} B-L$ model}

In the previous section, we have seen the difficulty of realizing the $\mathrm{CMB}$ anisotropy by QCD axion in the original $C C B-L$ model due to the smallness of the Higgs vev at the time of QCD phase transition. In this section, we discuss a possibility to overcome this situation by utilizing

${ }^{1}$ As long as

$$
g_{B-L}>\frac{\sqrt{3}}{16 \pi} \frac{m_{Z^{\prime}}^{3}}{\Lambda_{\mathrm{QCD}} m_{H} M_{p l}} \sim\left(\frac{m_{Z^{\prime}}}{10 \mathrm{PeV}}\right)^{3},
$$

the slow-roll condition at $\langle h\rangle=v_{\mathrm{QCD}}$ is violated, so that $\phi$ starts rolling down toward the true minimum as soon as the temperature gets to $T_{\text {end }}$. 
the idea of the symmetry nonrestoration (SNR) or inverse symmetry breaking (ISB) mechanism [35].

We extend the model by adding a gauge singlet $\mathcal{O}(N)$ scalar field $S$;

$$
\mathcal{L}=\mathcal{L}_{B-L}+\frac{1}{2}(\partial S)^{2}-V(S, H, \Phi) .
$$

Scalar potential is given by

$$
\begin{aligned}
V(S, H, \Phi)= & \frac{m_{S}^{2}}{2} S^{2}+\frac{\lambda_{S}}{4 !}\left(S^{2}\right)^{2}-\frac{\lambda_{S H}}{2} S^{2}\left(H^{\dagger} H\right) \\
& +\frac{\lambda_{S \phi}}{2} S^{2}\left(\Phi^{\dagger} \Phi\right),
\end{aligned}
$$

where $\mathcal{L}_{B-L}$ is the Lagrangian of the $B-L$ model with a QCD axion and $S^{2}:=\sum_{i=1}^{N} S_{i}^{2}$. In the following, we call a total set of SM, $B-L$ and QCD axion field as the $S M$ sector to distinguish $S$ fields as the $S$ sector.

Usually, the thermal mass of the SM Higgs, denoted by $\Pi_{h}$, is positive because of the large positive contribution from the top-loop $\sim y_{t}^{2} T^{2}$. However, as discussed in Refs. [35-42], it is also possible to obtain a negative effective mass through a negative Higgs portal coupling $-\lambda_{S H}$. Qualitatively, such a contribution is given by $\Delta \Pi_{h} \sim$ $-N \lambda_{S H} \tilde{T}^{2}$, where $\tilde{T}$ is the temperature of the $S$ field. In the following, we use $\tilde{T}$ to denote the temperature of the $S$ sector while $T$ without a tilde denotes the temperature of the SM sector. It indicates that $\Pi_{h}$ can be negative when the combination

$$
\tilde{\lambda}_{S H}:=N \lambda_{S H}
$$

is sufficiently large. Apparently, the behavior of the Higgs thermal mass can significantly change the thermal history of the universe and have various cosmological implications. See Refs. [36-42] for recent studies. It is usually assumed that $O(N)$ scalars $S$ are in the thermal equilibrium at the same temperature as the SM particles, $\tilde{T}=T$. For our present purpose, however, it is not desirable since all the energy scale is then given by the QCD scale at $\tilde{T}=T \sim$ $T_{\mathrm{QCD}}$ and the resultant Higgs vev would be also given by the same scale. Hence it is difficult to obtain $\langle h\rangle \sim$ $100 \mathrm{GeV}$ at $T \sim T_{\mathrm{QCD}}$.

In order to achieve a large Higgs vev, we will consider a different thermal history in which $S$ evolves almost separately from the SM sector in the early universe. If the temperature (or density) of $S$ is much higher than that of the SM sector, $\tilde{T} \gg T$, we can have a large negative contribution to the effective Higgs mass, and consequently $\langle h\rangle \sim 100 \mathrm{GeV}$ even at the QCD phase transition.

Based on the idea, we consider the following cosmological history of Eq. (33):

(1) $O(N)$ scalars $S$ are supposed to be dominantly produced in the primordial reheating and dominates the energy density of the universe until thermal inflation starts. We represent the energy densities and temperatures of $(S, \mathrm{SM})$ sectors as $\left(\rho_{S}, \rho_{\mathrm{SM}}\right)$ and $(\tilde{T}, T)$ respectively.

(2) Production processes $S S \rightarrow h h$ and $\phi \phi$ are assumed to be very tiny so that the temperature of two sectors evolve differently. The condition is guaranteed as long as the scalar mixings, $\lambda_{S H}, \lambda_{S \phi}$, are sufficiently small.

(3) At $T=T_{\mathrm{QCD}} \sim 150 \mathrm{MeV}$, the axion acquires potential and the primordial fluctuation of the axion field is converted to the fluctuations of the SM radiation. The temperature of the $S$ sector is denoted by $\tilde{T}_{\mathrm{QCD}}$ at this moment. After that, the production of the SM radiation from the $O(N)$ scalar should be ineffective so that the fluctuations are not diluted.

(4) Thermal inflation starts at $\tilde{T}=\tilde{T}_{\mathrm{TI}}$ (and $T=T_{\mathrm{TI}}$ ) when the dominant radiation energy $\rho_{S}$ in the early universe becomes comparable to $V_{\mathrm{TI}}$ of Eq. (18), and dilutes the initial abundance of $S$.

(5) Thermal inflation ends when the trapped field $\phi$ starts rolling down at the temperature $\tilde{T}=$ $\tilde{T}_{\text {end }}\left(T=T_{\text {end }}\right)$.

(6) Vacuum energy of the thermal inflation reheats up the SM sector. During and after the secondary reheating, the production of $S$ is required to be suppressed. The universe then follows the usual thermal history.

Figure 3 is a schematic picture of the thermal history of the $O(N)$ extended model. The thermal history of our model is highly dependent on the mass scale of $S$ and we will study two extremal cases, $m_{S} \gg m_{Z^{\prime}}$ and $m_{S}=0$. As we see, a viable model needs to satisfy the following two requirements:

(i) a requirement of large Higgs vev $\langle h\rangle \sim 246 \mathrm{GeV}$ at $T=T_{\mathrm{QCD}}$ gives a constraint, Eq. (61) for a massive

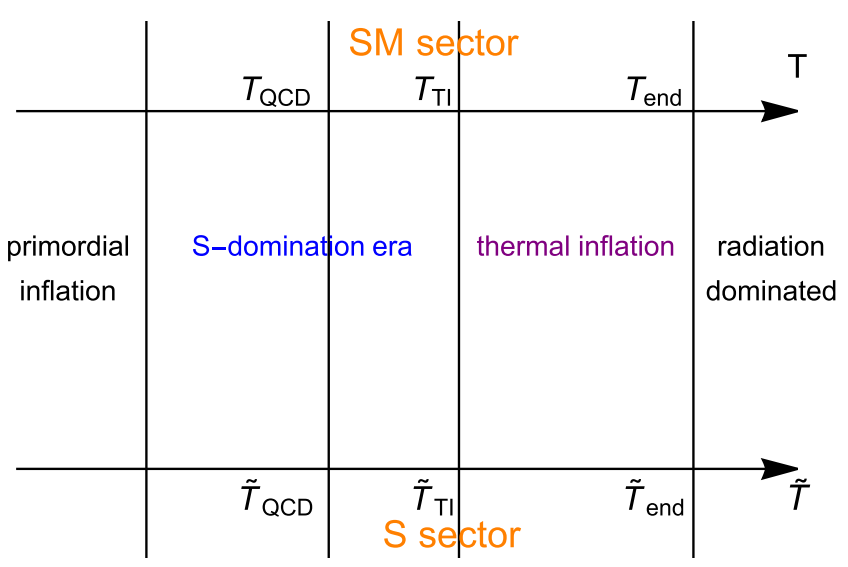

FIG. 3. Thermal history of the early universe of the AxionCMB scenario in the $O(N)$ extended model. The $O(N)$ sector evolves with different temperature $\tilde{T}$ from the SM sector with temperature $T$. 
case or Eq. (91) for a massless case, among various parameters of the model.

(ii) a requirement of sufficient dilutions of $S$ and axions during thermal inflation gives additional constraints on the model.

As we see in the following sections, we will find that only the massless case can be consistent with all the theoretical and observational constraints for $N \gtrsim 10^{19}$ and $m_{Z^{\prime}}=\mathcal{O}(10 \mathrm{TeV})$.

\section{Massive $O(N)$ sector with $m_{S} \geq m_{Z^{\prime}}$}

We first study details of the thermal history in the massive $S$ case. Although we see that the massive case does not have an allowed parameter region for the axionCMB scenario, we will show the detailed calculations for comparison to the massless case. We assume $m_{S} \geq m_{Z^{\prime}}$ to forbid reproduction of $S$ after the $B-L$ thermal inflation. As depicted in Fig. 3, the cosmological history can be divided into three different eras: matter dominated era by $O(N)$ scalar, thermal inflation era, and then the ordinary SM radiation dominated era follows the reheating after the thermal inflation.

\section{Evolution of $\left\langle S^{2}\right\rangle$ and $\langle h\rangle$}

We assume that the energy density that has driven the primordial inflation is converted only to the $O(N)$ scalar particle $S$, not to the SM sector particles. The initial distribution function of $S$ is set when $S$ becomes decoupled from other unspecified fields such as inflatons, and given by

$$
f_{S, \mathrm{dec}}(k)=\left(e^{\left(E_{k}-\mu_{\mathrm{dec}}\right) / \tilde{T}_{\mathrm{dec}}}-1\right)^{-1} \simeq e^{\left(\mu_{\mathrm{dec}}-E_{k}\right) / \tilde{T}_{\mathrm{dec}}},
$$

where $E_{k}=\sqrt{m_{S}^{2}+\left(k / a_{\mathrm{dec}}\right)^{2}}$ and we have assumed $m_{S}-$ $\mu_{\mathrm{dec}} \gg \tilde{T}_{\mathrm{dec}}$ for the Boltzmann approximation. After the decoupling, the number density is given by

$$
n_{S}=\frac{N}{a^{3}} \int \frac{d^{3} k}{(2 \pi)^{3}} f_{S, \operatorname{dec}}(k) \simeq N \alpha\left(\frac{m_{S} \tilde{T}}{2 \pi}\right)^{3 / 2},
$$

where we have defined $\alpha:=e^{\left(\mu_{\mathrm{dec}}-m_{S}\right) / \tilde{T}_{\mathrm{dec}}}$ and the effective temperature decreases as $\tilde{T}:=\tilde{T}_{\mathrm{dec}}\left(a_{\mathrm{dec}} / a\right)^{2}$. The number density $n_{S}$ and the energy density $\rho_{S} \simeq m_{S} n_{S}$ are diluted as $n_{S}, \rho_{S} \propto a^{-3}$.

Thermal inflation starts at $t=t_{\mathrm{TI}}$ when the energy density of $S$, which has dominated until then, becomes identical to the vacuum energy $V_{\mathrm{TI}}$ of the $B-L$ field,

$$
\rho_{S} \simeq m_{S} n_{S}=V_{\mathrm{TI}}
$$

Equation (36) tells us that the effective temperature of the $S$ sector at this moment is

$$
\tilde{T}_{\mathrm{TI}}=\frac{2 \pi}{m_{S}^{5 / 3}(\alpha N)^{2 / 3}} V_{\mathrm{TI}}^{2 / 3} \sim 0.013 \mathrm{GeV}\left(\frac{m_{Z^{\prime}}}{3 \times 10^{9} \mathrm{GeV}}\right)\left(\frac{4 \times 10^{15}}{\alpha N}\right)^{2 / 3}\left(\frac{m_{Z^{\prime}}}{m_{S}}\right)^{5 / 3} .
$$

We denote the SM energy density at $t=t_{\mathrm{TI}}$ as

$$
\rho_{\mathrm{SM}, \mathrm{TI}}:=\frac{\pi^{2} g_{\mathrm{SM}}}{30} T_{\mathrm{TI}}^{4},
$$

where $T_{\mathrm{TI}}$ is calculated below. In the following, we set the scale factor as $a=1$ at $t=t_{\mathrm{TI}}$. Then the Hubble parameter evolves as

$$
H=H_{\mathrm{TI}} \times \begin{cases}a^{-3 / 2} & \text { for } a \leq 1, \text { massive } S \text { dominated } \\ 1 & \text { for } a>1, \text { until the end of thermal inflation }\end{cases}
$$

where $H_{\mathrm{TI}}$ is defined by Eq. (25). The scale factor at the decoupling is given by

$$
a_{\mathrm{dec}}=\left(\tilde{T}_{\mathrm{TI}} / \tilde{T}_{\mathrm{dec}}\right)^{1 / 2},
$$

which becomes very small if decoupling occurs much earlier than the start of the thermal inflation. Since $S$ is massive and behaves nonrelativistically, the energy density $\rho_{S}$ is written in terms of the averaged amplitude of fluctuations as

$$
\rho_{S} \simeq m_{S}^{2}\left\langle S^{2}\right\rangle
$$

Thus we have

$$
\left\langle S^{2}\right\rangle=\frac{V_{\mathrm{TI}}}{m_{S}^{2}} a^{-3}=\frac{3 m_{Z^{\prime}}^{2}}{128 \pi^{2}}\left(\frac{m_{Z^{\prime}}}{m_{S}}\right)^{2} a^{-3}
$$

where Eq. (18) is used in the last equality.

Through the portal coupling $\lambda_{S H}$, the Higgs also acquires time-dependent vev in the early universe as 


$$
\langle h\rangle=\sqrt{\frac{\lambda_{S H}}{2 \lambda_{H}}\left\langle S^{2}\right\rangle} \sim \frac{3^{1 / 2} m_{Z^{\prime}}}{16 \pi} \sqrt{\frac{\lambda_{S H}}{\lambda_{H}}}\left(\frac{m_{Z^{\prime}}}{m_{S}}\right) a^{-3 / 2} .
$$

\section{Evolution of $\tilde{T}$}

It is useful to rewrite $\rho_{S}$ as a function of $\langle h\rangle$ by eliminating $\left\langle S^{2}\right\rangle$ from the above equations,

$$
\rho_{S}=\frac{2 \lambda_{H} m_{S}^{2}\langle h\rangle^{2}}{\lambda_{S H}} .
$$

By equating this to $m_{S} n_{S}$ with Eq. (36), the evolution of the temperature $\tilde{T}$ can be written in terms of $\langle h\rangle$ as

$$
\begin{aligned}
\tilde{T}= & 2 \pi\left(\frac{2 \lambda_{H}}{\alpha \tilde{\lambda}_{S H}}\right)^{2 / 3} \frac{\langle h\rangle^{4 / 3}}{m_{S}^{1 / 3}} \\
= & 2.3 \times 10^{4} \mathrm{GeV}\left(\frac{\lambda_{H}}{0.1}\right)^{2 / 3}\left(\frac{10^{-6}}{\alpha \tilde{\lambda}_{S H}}\right)^{2 / 3} \\
& \times\left(\frac{3 \times 10^{9} \mathrm{GeV}}{m_{S}}\right)^{1 / 3}\left(\frac{\langle h\rangle}{246 \mathrm{GeV}}\right)^{4 / 3} .
\end{aligned}
$$

See the schematic picture in Fig. 3 and the lower panel of Fig. 4. The induced Higgs mass via $\left\langle S^{2}\right\rangle$ is given by $m_{H, \text { eff }}=\sqrt{\lambda_{S H}\left\langle S^{2}\right\rangle}$. Then the ratio of $m_{S}$ to this becomes

$$
\left(\frac{m_{S}}{m_{H, \text { eff }}}\right)^{2}=\frac{1}{\tilde{\lambda}_{S H} \alpha}\left(\frac{2 \pi m_{S}}{T}\right)^{3 / 2} \gg 1
$$

and thus $h h \rightarrow S S$ is kinematically suppressed so that we can safely neglect the process.

\section{Production of SM particles and evolution of $X=\rho_{\mathrm{SM}} / \rho_{S}$}

The SM radiation $\rho_{\mathrm{SM}}$ is generated by the $S S \rightarrow H H$ process with the weak $\lambda_{S H}$ coupling. Let us now study its time evolution. Our assumption is that the process is extremely low and the ratio

$$
X:=\rho_{\mathrm{SM}} / \rho_{S}
$$

is much less that unity until the thermal inflation. Under this assumption, we can safely neglect the back-reaction to $\rho_{S}$ and the behavior $\rho_{S} \propto a^{-3}$ discussed above remains intact. Then the Boltzmann equation of $\rho_{\mathrm{SM}}$ is given by
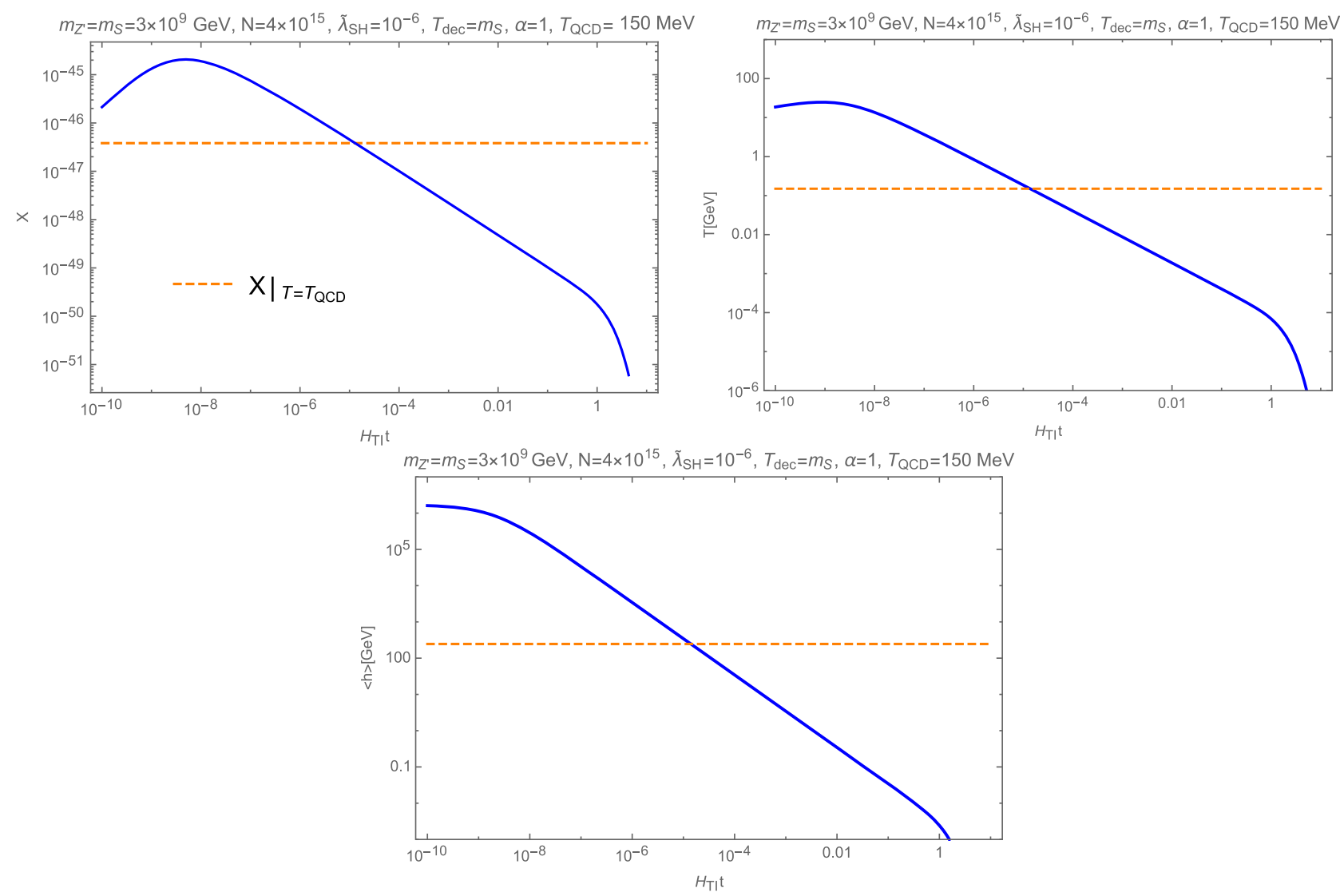

FIG. 4. Time evolutions of the ratio $X=\rho_{\mathrm{SM}} / \rho_{S}$ (top-left), temperature of the SM sector $T$ (top-right) and the Higgs vev (down). The orange line corresponds to the value of each quantity at $T=T_{\mathrm{QCD}}$. 


$$
\dot{\rho}_{\mathrm{SM}}+4 H \rho_{\mathrm{SM}} \simeq m_{S} \frac{\lambda_{S H}^{2}}{16 \pi m_{S}^{2}} n_{S} n_{i}=\frac{\lambda_{S H}^{2}}{16 \pi m_{S}^{3}} \rho_{S} \rho_{i}
$$

where $n_{i}:=n_{S} / N$ and $\rho_{i}:=\rho_{S} / N$. Its derivation is presented in A. From this, the ratio (48) obeys the following equation;

$$
\frac{d X}{d t}+H X=\frac{\lambda_{S H}^{2}}{16 \pi N m_{S}^{3}} \rho_{S}=\frac{\lambda_{S H}^{2} V_{\mathrm{TI}}}{16 \pi N m_{S}^{3}} a^{-3} .
$$

Inserting the explicit time-dependence of $\rho_{S}$ and the Hubble parameter $H=d \ln a / d t$, we have

$$
\frac{d(a X)}{d a}=\frac{\lambda_{S H}^{2} V_{\mathrm{TI}}}{16 \pi N m_{S}^{3} H} a^{-3}:=c \times\left\{\begin{array}{ll}
a^{-3 / 2} & \text { for } a \leq 1 \\
a^{-3} & \text { for } a>1
\end{array},\right.
$$

where

$$
c:=\frac{\sqrt{3} \tilde{\lambda}_{S H}^{2} M_{p l} V_{\mathrm{TI}}^{1 / 2}}{16 \pi N^{3} m_{S}^{3}} .
$$

It can be integrated as (noting $a_{\mathrm{TI}}=1$ )

$$
a X-X_{\mathrm{TI}}=c \times\left\{\begin{array}{ll}
2\left(1-a^{-1 / 2}\right) & \text { for } a \leq 1 \\
\frac{1}{2}\left(1-a^{-2}\right) & \text { for } a>1
\end{array},\right.
$$

where $X_{\mathrm{TI}}$ is the value of $X$ at $T=T_{\mathrm{TI}}$. By denoting the initial value of $X$ right after the decoupling of $S$ as $X_{\mathrm{dec}}$, we have

$X_{\mathrm{TI}}=a_{\mathrm{dec}} X_{\mathrm{dec}}-2 c\left(1-a_{\mathrm{dec}}^{-1 / 2}\right) \simeq a_{\mathrm{dec}} X_{\mathrm{dec}}+2 c a_{\mathrm{dec}}^{-1 / 2}$.

The top-left figure of Fig. 4 shows the time evolution of the ratio with $X_{\mathrm{dec}}=0$. It first grows with time through the energy transfer from $S$ and SM sectors. At $a \sim\left(3 c / X_{\mathrm{TI}}\right)^{2} \sim(3 / 2)^{2} a_{\mathrm{dec}} \ll 1$, it starts decreasing as $X \sim a^{-1}$ since the $S S \rightarrow H H$ process is not fast enough to overwhelm the expansion and $\rho_{\mathrm{SM}} \sim a^{-4}$ is diluted more quickly than $\rho_{S} \sim a^{-3}$ is. This behavior continues in the thermal inflation period;

$$
X \rightarrow \frac{1}{a}\left(X_{\mathrm{TI}}+\frac{c}{2}\right) \sim \frac{X_{\mathrm{TI}}}{a} \text { for } a \gg 1 .
$$

In the following, it is supposed that the first term $a_{\mathrm{dec}} X_{\mathrm{dec}}$ in Eq. (54), which depends on the unspecified dynamics before the decoupling, is negligible. The initial conditions of later evolution are given by $\tilde{T}_{\mathrm{dec}}$ and $\mu_{\mathrm{dec}}$, which characterize the physics in the primordial reheating era.

\section{Various quantities at $T=T_{\mathrm{QCD}}$}

The top-right figure of Fig. 4 is the temperature evolution of the SM sector, $T$. It first grows by the energy transfer from $S$ sector, but then decreases due to the expansion of the universe and eventually reaches $T_{\mathrm{QCD}}$, which is depicted by the orange line. The scale factor at which $T$ gets down to $T_{\mathrm{QCD}} \sim 150 \mathrm{MeV}$ is evaluated as

$$
\begin{gathered}
a_{\mathrm{QCD}}=\left(\frac{\rho_{\mathrm{SM}, \mathrm{TI}}}{\rho_{\mathrm{SM}, \mathrm{QCD}}}\right)^{1 / 4}=\left(\frac{X_{\mathrm{TI}} V_{\mathrm{TI}}}{\rho_{\mathrm{SM}, \mathrm{QCD}}}\right)^{1 / 4} \\
=7.6 \times 10^{-4} \times \alpha^{\frac{1}{24}}\left(\frac{\tilde{\lambda}_{S H}}{10^{-6}}\right)^{\frac{1}{2}}\left(\frac{4 \times 10^{15}}{N}\right)^{17 / 24} \\
\times\left(\frac{100}{g_{\mathrm{SM}}}\right)^{1 / 4}\left(\frac{150 \mathrm{MeV}}{T_{\mathrm{QCD}}}\right) \\
\times\left(\frac{m_{Z^{\prime}}}{3 \times 10^{9} \mathrm{GeV}}\right)^{\frac{3}{4}}\left(\frac{m_{Z^{\prime}}}{m_{S}}\right)^{\frac{7}{12}}\left(\frac{\tilde{T}_{\mathrm{dec}}}{m_{S}}\right)^{1 / 16}
\end{gathered}
$$

where we used $\rho_{\mathrm{SM}, \mathrm{QCD}}=\left(g_{\mathrm{SM}} \pi^{2} / 30\right) T_{\mathrm{QCD}}^{4}$. From Eq. (57), we can confirm $\tilde{T}_{\mathrm{QCD}}=\tilde{T}_{\mathrm{TI}} a_{\mathrm{QCD}}^{-1} \gg \tilde{T}_{\mathrm{TI}}$ again. As mentioned after Eq. (46), $T=T_{\mathrm{QCD}}$ is realized well before the beginning of thermal inflation. Note also that the SM temperature $T_{\mathrm{TI}}$ satisfies

$$
T_{\mathrm{TI}}=T_{\mathrm{QCD}} a_{\mathrm{QCD}} \ll T_{\mathrm{QCD}} .
$$

Recall that we need $\langle h\rangle=\mathcal{O}(100 \mathrm{GeV})$ at $T=T_{\mathrm{QCD}}$ for the axion-CMB scenario to be observationally viable in the CC $B-L$ model. The time evolution of $\langle h\rangle$ is plotted in the down figure of Fig. 4. From Eqs. (45) and (48), the condition for the Higgs vev is written as

$$
\left.X\right|_{T=T_{\mathrm{QCD}}}=\frac{\lambda_{S H} \rho_{\mathrm{SM}, \mathrm{QCD}}}{\left.2 \lambda_{H} m_{S}^{2}\langle h\rangle^{2}\right|_{T=T_{\mathrm{QCD}}}} .
$$

On the other hand, from Eqs. (55) and (56), we have

$$
\left.X\right|_{T=T_{\mathrm{QCD}}}=X_{\mathrm{TI}}^{3 / 4}\left(\frac{\rho_{\mathrm{SM}, \mathrm{QCD}}}{V_{\mathrm{TI}}}\right)^{1 / 4} .
$$

By equating Eq. (60) to Eq. (59), $N$ is determined in terms of other parameters of the model;

$$
\begin{aligned}
N \sim & \times 10^{15} \times\left(\frac{\tilde{\lambda}_{S H}}{10^{-6}}\right)^{4 / 9}\left(\frac{\lambda_{H}}{0.1}\right)^{8 / 9}\left(\frac{100}{g_{\mathrm{SM}}}\right)^{2 / 3} \\
& \times\left(\frac{\langle h\rangle}{246 \mathrm{GeV}}\right)^{16 / 9}\left(\frac{150 \mathrm{MeV}}{T_{\mathrm{QCD}}}\right)^{8 / 3} \\
& \times\left(\frac{m_{S}}{3 \times 10^{9} \mathrm{GeV}}\right)^{2 / 9}\left(\frac{T_{\mathrm{dec}}}{m_{S}}\right)^{1 / 6} .
\end{aligned}
$$

As we discussed in Eq. (5), the Higgs must have as large vev at $T=T_{\mathrm{QCD}}$ as the current value so that it is consistent 
with the non-Gaussianity constraint and the CMB amplitude can be explained in terms of the primordial axion fluctuations. Then, we see from the above relation, that $N$ must be quite large.

Finally note that the assumption $a_{\mathrm{dec}} \ll 1$ is justified as far as

$$
\left(\frac{T_{\mathrm{dec}}}{m_{S}}\right)^{3 / 2} \alpha \times N \gg(2 \pi)^{3 / 2} \frac{V_{\mathrm{TI}}}{m_{S}^{4}} .
$$

\section{Dilution factor by thermal inflation}

By definition, the number density of $S$ at $\tilde{T}=\tilde{T}_{\mathrm{TI}}$ is $V_{\mathrm{TI}} / m_{S}$ and it is diluted rapidly during the thermal inflation. The Higgs vev $\langle h\rangle$ decreases and finally approaches the value $v_{\mathrm{QCD}}$ determined by the QCD chiral condensates in Eq. (27);

$$
\langle h\rangle^{2}=\left\{\begin{array}{ll}
\left.\langle h\rangle^{2}\right|_{T=T_{\mathrm{QCD}}}\left(a_{\mathrm{QCD}} / a\right)^{3} & T \sim T_{\mathrm{QCD}} \\
v_{\mathrm{QCD}}^{2} & T \ll T_{\mathrm{QCD}}
\end{array} .\right.
$$

where $a_{\mathrm{QCD}}$ is given by Eq. (57).

To calculate the dilution factor, we need to know the temperature $T_{\text {end }}$ at which the thermal inflation ends. In presence of the $S$ sector, the quadratic term of the $B-L$ scalar $\phi$ of Eq. (28) is modified to be

$$
\frac{T^{2}}{8} M_{Z^{\prime}}^{2}(\phi)-\frac{\lambda_{\phi H}}{4}\langle h\rangle^{2} \phi^{2}+\frac{\tilde{\lambda}_{S \phi}}{4 N}\left\langle S^{2}\right\rangle \phi^{2} .
$$

For simplicity, we consider a situation where the last term is negligibly small by assuming the smallness of $\tilde{\lambda}_{S \phi}$,

$$
\frac{\tilde{\lambda}_{S \phi}}{4 N}\left\langle S^{2}\right\rangle=\frac{\tilde{\lambda}_{S \phi} \lambda_{H}}{2 \tilde{\lambda}_{S H}}\langle h\rangle^{2} \ll \frac{\lambda_{\phi H}}{4}\langle h\rangle^{2},
$$

where we have used Eq. (44). Then the end of the thermal inflation is determined by the first two terms of Eq. (64);

$$
\begin{aligned}
\frac{T^{2}}{2} & g_{B-L}^{2} \phi^{2}-g_{B-L}^{2}\left(\frac{m_{h}}{m_{Z^{\prime}}}\right)^{2}\langle h\rangle^{2} \phi^{2} \\
& =g_{B-L}^{2}\left[\frac{T^{2}}{2}-\left(\frac{m_{H}}{m_{Z^{\prime}}}\right)^{2}\langle h\rangle^{2}\right] \phi^{2} .
\end{aligned}
$$

As long as $\langle h\rangle^{2}$ decreases as $a^{-3}$ [see Eq. (63)], the first term in Eq. (66) is always dominant because $T^{2}$ decreases as $a^{-2}$. Thus, these two terms can become comparable after $\langle h\rangle$ reaches the constant value $v_{\mathrm{QCD}}$, which means that $T_{\text {end }}$ is similarly determined as in the case of the conventional $B-L$ model and given by Eq. (30). Then the dilution factor during the thermal inflation from $T=T_{\mathrm{TI}}$ until $T=$ $T_{\text {end }}$ is obtained as $e^{-3 \Delta N}=\left(\frac{T_{\mathrm{end}}}{T_{\mathrm{TI}}}\right)^{3}=\frac{1}{2^{3 / 2}}\left(\frac{v_{\mathrm{QCD}}}{T_{\mathrm{QCD}}}\right)^{3}\left(\frac{m_{H}}{m_{\mathrm{Z}^{\prime}}}\right)^{3} \times a_{\mathrm{QCD}}^{-3}$.

If $m_{Z^{\prime}}$ is sufficiently heavy, the dilution factor during the thermal inflation becomes large.

\section{Dilution of axions}

We now estimate $r_{A}$ in the massive case. The axion field starts to oscillate when $m_{A}(T) \geq 3 H$ is satisfied. Here, $m_{A}(T)$ is the temperature-dependent axion mass:

$$
m_{A}(T)=m_{A 0} \times\left\{\begin{array}{ll}
\left(T_{\mathrm{QCD}} / T\right)^{4 b} & \text { for } T \geq T_{\mathrm{QCD}} \\
1 & \text { for } T \leq T_{\mathrm{QCD}}
\end{array},\right.
$$

where $b \sim 1.02$. In the massive $S$ case, the solution is determined by $m_{A 0}=3 H$;

$$
\begin{aligned}
\tilde{T}_{\text {osc }}= & \frac{2 \pi\left(M_{p l} m_{A 0}\right)^{4 / 3}}{3^{2 / 3}(\alpha N)^{2 / 3} m_{S}^{5 / 3}} \\
= & 6.9 \times 10^{-21} \mathrm{GeV}\left(\frac{4 \times 10^{15}}{\alpha N}\right)^{2 / 3}\left(\frac{3 \times 10^{9} \mathrm{GeV}}{m_{S}}\right)^{5 / 3} \\
& \times\left(\frac{m_{A 0}}{6 \times 10^{-6} \mathrm{eV}}\right)^{4 / 3}
\end{aligned}
$$

which is actually tiny compared to $\tilde{T}_{\mathrm{QCD}}$ and $\tilde{T}_{\mathrm{TI}}$, which is consistent with the assumption $T_{\text {osc }}<T_{\mathrm{QCD}}$. Thus, the axion field does not evolve until the beginning of thermal inflation and we can use the same result of $r_{A}$ calculated in Ref. [15] with a different $e$-folding number;

$r_{A} \sim\left(\frac{2 \tan \left(\bar{\theta}_{\text {ini }} / 2\right)}{0.3}\right)^{2}\left(\frac{f_{A}}{10^{12} \mathrm{GeV}}\right)^{1.16} \exp (-2 \eta \times \Delta N)$,

where

$\eta:=\frac{1}{3}\left(\frac{m_{A 0}}{H_{\mathrm{TI}}}\right)^{2}=8.6 \times 10^{-6} \times\left(\frac{10^{12} \mathrm{GeV}}{f_{A}}\right)^{2}\left(\frac{10 \mathrm{TeV}}{m_{Z^{\prime}}}\right)^{4}$

and $\Delta N=\log \left(T_{\mathrm{TI}} / T_{\text {end }}\right)$. The result (70) shows that, in the massive case, $Z^{\prime}$ gauge boson has to be lighter than $10 \mathrm{TeV}$ for realizing the necessary condition of $r_{A} \lesssim 10^{-4}$. On the other hand, as we see in the following, a condition for sufficient dilution of $S$ requires $m_{Z^{\prime}} \gg 10 \mathrm{TeV}$. Thus it is impossible to satisfy all the observational constraints in the massive $S$ case. 


\section{Dilution of initial abundance of $S$}

In addition to the dilution of the axion abundance, the thermal inflation also has to dilute the initial abundance of $S$. Otherwise, such an abundance may cause various cosmological problems. Especially $r_{S}:=\rho_{S} / \rho_{\mathrm{DM}}$ must satisfy the same constraint as in Eq. (15),

$$
r_{S}<8.2 \times 10^{-3} R \text {. }
$$

Let us now evaluate the relic abundance of $S$ at the present universe. After the thermal inflation ends at $a=a_{\text {end }}$, the energy density of the $\phi$-oscillation dominates the universe until the universe is reheated to the temperature $T_{R}$. The relic abundance of $S$ is estimated as

$$
\begin{aligned}
\left.\rho_{S}\right|_{\text {today }} & \sim\left(\frac{a_{\text {end }}}{a_{\text {today }}}\right)^{3} e^{-3 \Delta N} \times V_{\mathrm{TI}} \\
& \sim \frac{g_{R} \pi^{2}}{30} \frac{T_{R}^{4}}{V_{\mathrm{TI}}}\left(\frac{T_{\text {today }}}{T_{R}}\right)^{3} e^{-3 \Delta N} \times V_{\mathrm{TI}},
\end{aligned}
$$

where $T_{\text {today }}=2.73 \mathrm{~K}$ and $g_{R}$ is the effective degrees of freedom at the reheating. By substituting Eq. (67) into Eq. (73), we obtain

$$
\begin{aligned}
r_{S}:= & \rho_{S} /\left.\rho_{\mathrm{DM}}\right|_{\text {today }}=\left.\left(\rho_{S} / \rho_{\gamma}\right)\left(\rho_{\gamma} / \rho_{\mathrm{DM}}\right)\right|_{\text {today }} \\
= & 0.011 \times\left(\frac{10^{-6}}{\tilde{\lambda}_{S H}}\right)^{3 / 2}\left(\frac{N}{4 \times 10^{15}}\right)^{17 / 8}\left(\frac{v_{\mathrm{QCD}}}{T_{\mathrm{QCD}}}\right)^{3} \\
& \times\left(\frac{T_{\mathrm{QCD}}}{150 \mathrm{MeV}}\right)^{3}\left(\frac{10^{6} \mathrm{TeV}}{m_{Z^{\prime}}}\right)^{21 / 4}\left(\frac{m_{S}}{m_{Z^{\prime}}}\right)^{7 / 4} \\
& \times\left(\frac{T_{R}}{10 \mathrm{MeV}}\right)\left(\frac{m_{S}}{T_{\text {dec }}}\right)^{3 / 16},
\end{aligned}
$$

where we used $\left.\left(\rho_{\gamma} / \rho_{\text {DM }}\right)\right|_{\text {today }}=2.9 \times 10^{-4}$. Even with the reheating temperature around its lower limit $\sim 10 \mathrm{MeV}$ for the successful big bang nucleosynthesis, large $m_{Z^{\prime}}$ is required. Therefore, the two necessary conditions of $r_{A}, r_{S}<10^{-3}$ cannot be simultaneously satisfied.

\section{Massless $\boldsymbol{O}(\boldsymbol{N})$ sector}

In the previous subsection, we saw a difficulty to realize the axion-CMB scenario for $m_{S} \geq m_{Z^{\prime}}$. Here, let us consider another extremal case of $m_{S}=0$. The following discussion is almost parallel to the massive case except that (i) the SM production processes can be kinematically suppressed if $\tilde{\lambda}_{S H} \gtrsim 1$ and (ii) the SM radiation produced directly from the primordial reheating needs to be taken into account.

\section{Evolution of $\langle\boldsymbol{h}\rangle$}

The number and energy densities of $S$ after the primordial reheating are now given by

$$
n_{S}=\frac{N \zeta(3)}{\pi^{2}} \tilde{T}^{3}, \quad \rho_{S}=\frac{N \pi^{2}}{30} \tilde{T}^{4},
$$

where $\tilde{T}=\tilde{T}_{\text {reh }}\left(a_{\text {reh }} / a\right)$.

Thermal inflation starts at $t=t_{\mathrm{TI}}$ when $\rho_{S}$ becomes identical to the vacuum energy of the $B-L$ field, $\rho_{S}=V_{\mathrm{TI}}$. The corresponding temperature of the $S$ sector is

$$
\tilde{T}_{\mathrm{TI}}=\left(\frac{30}{\pi^{2} N}\right)^{1 / 4} V_{\mathrm{TI}}^{1 / 4} \sim 0.05 \mathrm{GeV} \times\left(\frac{m_{Z^{\prime}}}{10 \mathrm{TeV}}\right)\left(\frac{10^{19}}{N}\right)^{1 / 4} .
$$

Since the scale factor is set as $a=1$ at $t=t_{\mathrm{TI}}$, the Hubble parameter behaves as

$$
H=H_{\mathrm{TI}} \times \begin{cases}a^{-2} & \text { for } a \leq 1, \text { massless } S \text { dominated } \\ 1 & \text { for } a>1, \text { until the end of thermal inflation }\end{cases}
$$

where $H_{\mathrm{TI}}$ is defined by Eq. (25). In the massless case, the Higgs vev is determined by the thermal mass $-\tilde{\lambda}_{\mathrm{SH}} \tilde{T}^{2} / 24$ as in the conventional studies [35-38] and given by

$$
\langle h\rangle^{2}=\tilde{\lambda}_{S H} \tilde{T}^{2} /\left(24 \lambda_{H}\right) .
$$

We require that $\langle h\rangle \sim 246 \mathrm{GeV}$ at $T=T_{\mathrm{QCD}}$. Note also that, because of the Higgs vev, one of the components of $O(N)$ scalar acquires mass $\sqrt{\lambda_{S H}}\langle h\rangle \sim \tilde{\lambda}_{S H} \tilde{T} / \sqrt{N}$. However, it is much smaller than $\tilde{T}$ so that the massive component can be regarded as practically massless.

\section{Production of SM particles and evolution of $X=\rho_{\mathrm{SM}} / \rho_{S}$}

If $\lambda_{S H}<1, S S \rightarrow h h$ is the dominant process as in the massive case. On the other hand, for $\lambda_{S H} \gtrsim 1$, the thermal mass of the Higgs $\sim \sqrt{\tilde{\lambda}_{S H}} \tilde{T}$ becomes larger than the radiation temperature $\tilde{T}$ of the $O(N)$ scalar sector, and the SM production rate gets Boltzmann-suppressed. Then, by introducing the suppression factor $\epsilon$, the Boltzmann equation of $\rho_{\mathrm{SM}}$ is given by

$$
\begin{aligned}
\dot{\rho}_{\mathrm{SM}}+4 H \rho_{\mathrm{SM}} & =\frac{\epsilon \lambda_{S H}^{2}}{128 \pi \tilde{T}} n_{S} n_{i} \\
& =\frac{\epsilon \tilde{\lambda}_{S H}^{2}}{128 \pi N^{9 / 4}}\left(\frac{30}{\pi^{2}}\right)^{5 / 4}\left(\frac{\zeta(3)}{\pi^{2}}\right)^{2} \rho_{S}^{5 / 4},
\end{aligned}
$$

where we have used

$$
n_{S}=\frac{30 \zeta(3)}{\pi^{4}} \frac{\rho_{S}}{\tilde{T}}, \quad \tilde{T}=\left(\frac{30 \rho_{S}}{\pi^{2} N}\right)^{1 / 4} .
$$


The suppression factor $\epsilon \leq 1$ is normalized as $\epsilon=1$ when the process $S S \rightarrow h h$ is unsuppressed, and it dose not have any temperature dependence since there is no explicit mass scale. Note that SM particles other than Higgs are also produced; $S S \rightarrow$ (SM particles). A process like $S S \rightarrow b \bar{b}$ may not be thermally suppressed unlike $S S \rightarrow h h$, but it is negligibly small because of the tiny Yukawa coupling. For example we have $\epsilon \sim y_{b}^{2} \tilde{\lambda}_{S H}^{-1} \sim 10^{-5}$ for $\tilde{\lambda}_{S H} \sim 10$ and we can safely neglect them.

Then, the ratio $X=\rho_{\mathrm{SM}} / \rho_{S}$ obeys

$$
\begin{aligned}
\frac{d X}{d a} & =\frac{1}{a H} \frac{d X}{d t}=\frac{\epsilon \tilde{\lambda}_{S H}^{2}}{128 \pi N^{9 / 4}}\left(\frac{30}{\pi^{2}}\right)^{5 / 4}\left(\frac{\zeta(3)}{\pi^{2}}\right)^{2} \frac{\rho_{S}^{1 / 4}}{a H} \\
& =c \times\left\{\begin{array}{ll}
1 & \text { for } a \leq 1 \\
a^{-2} & \text { for } a \geq 1
\end{array},\right.
\end{aligned}
$$

where

$$
\begin{aligned}
c & =\frac{\epsilon \tilde{\lambda}_{S H}^{2}}{128 \pi N^{9 / 4}}\left(\frac{30}{\pi^{2}}\right)^{5 / 4}\left(\frac{\zeta(3)}{\pi^{2}}\right)^{2} \frac{V_{\mathrm{TI}}^{1 / 4}}{H_{\mathrm{TI}}} \\
& =\frac{3^{3 / 2} 5^{5 / 4} \zeta(3)^{2} \tilde{\lambda}_{S H}^{2}}{2^{4} \pi^{7} N^{9 / 4}} \frac{M_{p l}}{m_{Z^{\prime}}} .
\end{aligned}
$$

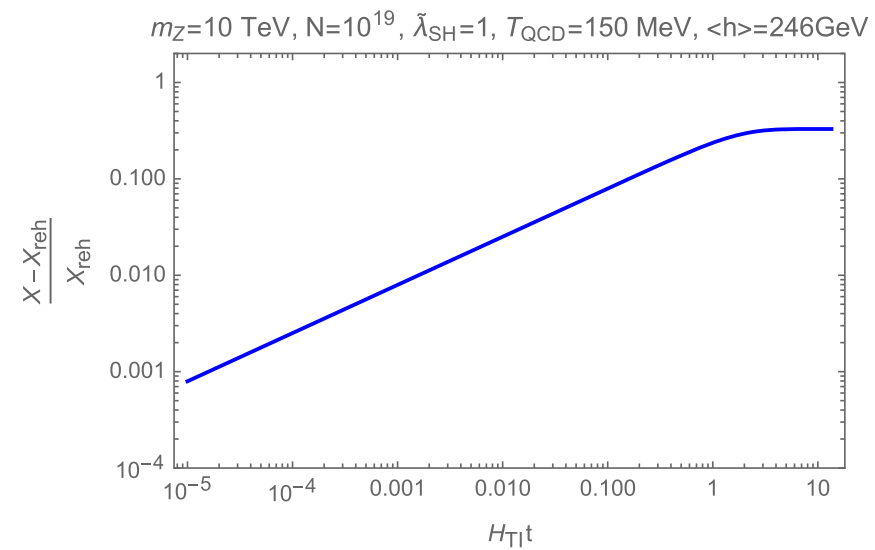

Equation (81) can be solved as

$$
X-X_{\mathrm{TI}}=c \times\left\{\begin{array}{ll}
a-1 & \text { for } a \leq 1 \\
1-a^{-1} & \text { for } a \geq 1
\end{array},\right.
$$

from which we obtain

$$
X_{\mathrm{TI}} \simeq X_{\text {reh }}+c \quad\left(\text { for } a_{\text {reh }} \ll 1\right)
$$

and

$$
X \simeq X_{\mathrm{TI}}+c \simeq X_{\text {reh }}+2 c \quad(\text { for } a \gg 1),
$$

where $X_{\text {reh }}$ comes from the SM energy density generated during the reheating after the primordial inflation. In the top-left panel of Fig. 5, we show the time evolution of $\left(X-X_{\text {reh }}\right) / X_{\text {reh. }}$. Note that, compared to the massive case Eq. (55), the ratio $X$ keeps increasing and approaches a constant value $X_{\text {reh }}+2 c$ because both of $S$ and SM particles behave as radiation and their energy densities dilute with $a^{-4}$. On the other hand, if $X_{\text {reh }}$ is larger than $2 c$,

$$
X_{\text {reh }}>2 c
$$

$X$ is almost constant from the onset of the $O(N)$-radiation dominated phase,
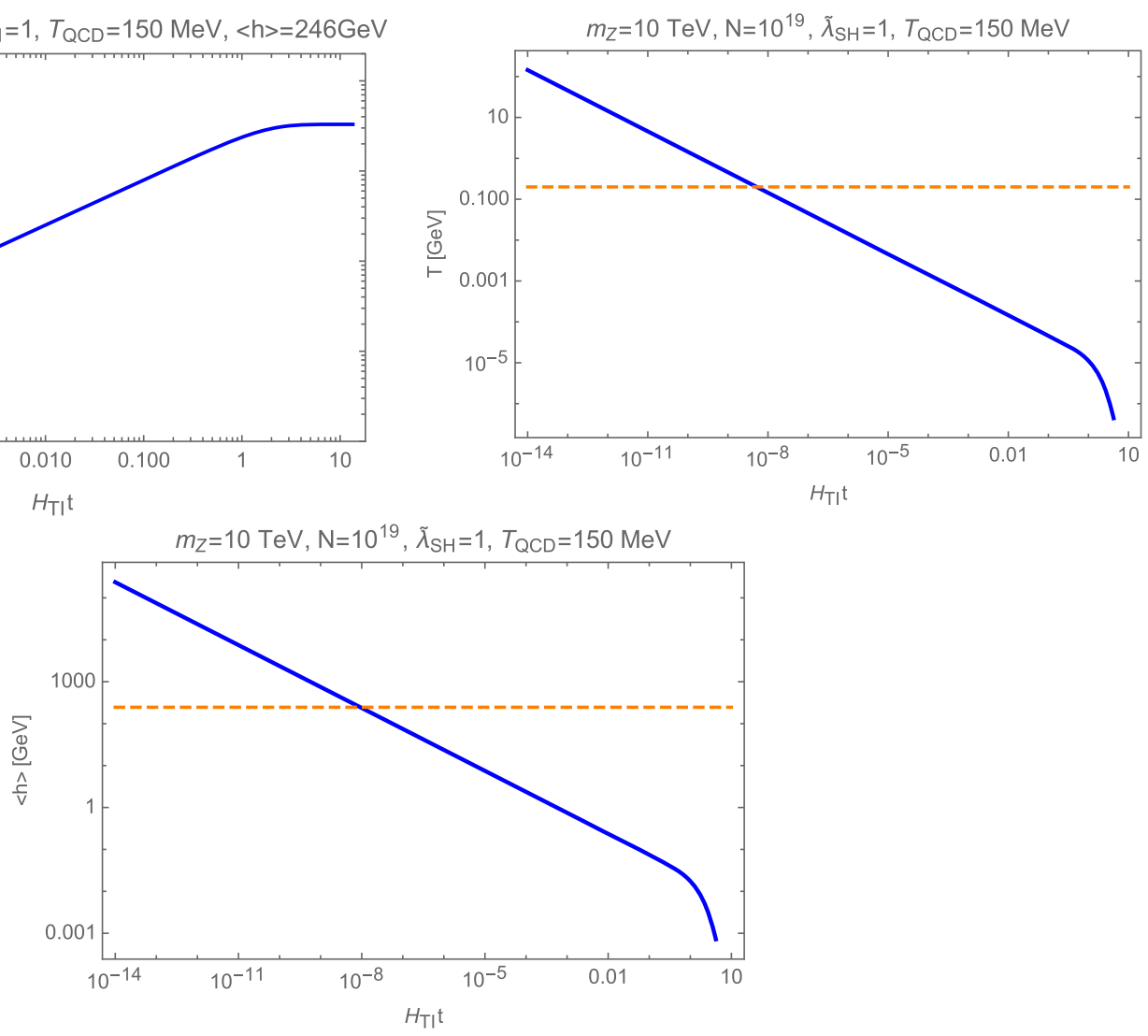

FIG. 5. Time evolutions of the ratio $X=\rho_{S} / \rho_{\mathrm{SM}}$ (top left), temperature of the SM sector $T$ (top right) and the Higgs vev (down) in the massless case. 


$$
X \simeq X_{\text {reh }}
$$

We will see that this situation is necessary for the axionCMB scenario in the massless $O(N)$ case because otherwise the $\mathrm{CMB}$ fluctuations transferred from the axions are diluted after QCD phase transition.

\section{Various quantities at $\boldsymbol{T}_{\mathrm{QCD}}$}

The required value of $N$ is determined in a similar way as the massive case. By using Eq. (78), the condition for the Higgs vev at $T=T_{\mathrm{QCD}}$ becomes

$$
\begin{aligned}
\left.X\right|_{T=T_{\mathrm{QCD}}} & =\left.\frac{\rho_{\mathrm{SM}}}{\rho_{S}}\right|_{T=T_{\mathrm{QCD}}}=\frac{g_{\mathrm{SM}}}{N}\left(\frac{T_{\mathrm{QCD}}}{\tilde{T}}\right)^{4} \\
& =\frac{g_{\mathrm{SM}}}{N}\left(\frac{\tilde{\lambda}_{S H}}{24 \lambda_{H}}\right)^{2}\left(\frac{T_{\mathrm{QCD}}}{\langle h\rangle}\right)^{4}
\end{aligned}
$$

Let $\tilde{T}_{\mathrm{QCD}}$ denote the temperature $\tilde{T}$ of $S$ sector at $T=T_{\mathrm{QCD}}$. The scale factor at this moment is then given by

$$
\begin{aligned}
a_{\mathrm{QCD}}:= & \left.a\right|_{T=T_{\mathrm{QCD}}}=\frac{\tilde{T}_{\mathrm{TI}}}{\tilde{T}_{\mathrm{QCD}}} \\
\sim & 1.4 \times 10^{-4} \times \tilde{\lambda}_{S H}^{1 / 2}\left(\frac{0.1}{\lambda_{H}}\right)^{1 / 2}\left(\frac{10^{19}}{N}\right)^{1 / 4} \\
& \times\left(\frac{m_{Z^{\prime}}}{10 \mathrm{TeV}}\right)\left(\frac{246 \mathrm{GeV}}{\langle h\rangle}\right),
\end{aligned}
$$

where we used Eqs. (76) and (78). Due to $H_{\mathrm{TI}} t_{\mathrm{QCD}}=$ $a_{\mathrm{QCD}}^{2} \sim 2 \times 10^{-8} \ll H_{\mathrm{TI}} t_{\mathrm{TI}}=1$, it is much before the beginning of the thermal inflation. This means that, if Eq. (86) is not satisfied, the perturbations of $\rho_{\text {SM }}$ generated at the QCD phase transition is diluted due to further productions of the SM particles from the $O(N)$ scalar after the transition. Hence, for realizing the axion-CMB scenario in explained in Sec. III, we simply impose the condition Eq. (86). Then $X$ is almost constant as in Eq. (87) and it must be given by

$$
X_{\mathrm{reh}}=\frac{g_{\mathrm{SM}}}{N}\left(\frac{\tilde{\lambda}_{S H}}{24 \lambda_{H}}\right)^{2}\left(\frac{T_{\mathrm{QCD}}}{\langle h\rangle}\right)^{4}
$$

to realize Eq. (88). Combined with Eq. (86), it reads

$$
\begin{aligned}
N> & 7.9 \times 10^{17}\left(\frac{\epsilon}{0.1}\right)^{4 / 5}\left(\frac{100}{g_{\mathrm{SM}}}\right)^{4 / 5}\left(\frac{10 \mathrm{TeV}}{m_{Z^{\prime}}}\right)^{4 / 5}\left(\frac{\lambda_{H}}{0.1}\right)^{8 / 5} \\
& \times\left(\frac{150 \mathrm{MeV}}{T_{\mathrm{QCD}}}\right)^{16 / 5}\left(\frac{\langle h\rangle}{246 \mathrm{GeV}}\right)^{16 / 5} .
\end{aligned}
$$

As mentioned in the Introduction, such a large value of $N$ is still allowed phenomenologically because collider observables are typically functions of $N \lambda_{S H}^{2}=\tilde{\lambda}_{S H}^{2} / N$ or $N \lambda_{S H}^{3}=\tilde{\lambda}_{S H}^{3} / N^{2}$. See Refs. [36,38] for more details.

\section{Dilution factor by thermal inflation}

First, $\rho_{S}$ is given by $V_{\mathrm{TI}}$ at $\tilde{T}=\tilde{T}_{\mathrm{TI}}$ by definition. The Higgs vev $\langle h\rangle$ decreases with temperature and finally approaches $v_{\mathrm{QCD}}$;

$$
\langle h\rangle^{2}=\left\{\begin{array}{ll}
\left.\langle h\rangle^{2}\right|_{T=T_{\mathrm{QCD}}}\left(a_{\mathrm{QCD}} / a\right)^{2} & T \sim T_{\mathrm{QCD}} \\
v_{\mathrm{QCD}}^{2} & T \ll T_{\mathrm{QCD}}
\end{array} .\right.
$$

We need to know the temperature $\tilde{T}_{\text {end }}$ at which the thermal inflation ends. In the massless case, the quadratic term of the $B-L$ scalar $\phi$ of Eq. (28) is modified to be

$$
\frac{T^{2}}{2} g_{B-L}^{2} \phi^{2}+\frac{\tilde{\lambda}_{S \phi}}{24} \tilde{T}^{2} \phi^{2}-g_{B-L}^{2}\left(\frac{m_{H}}{m_{Z^{\prime}}}\right)^{2}\langle h\rangle^{2} \phi^{2},
$$

where the second term is the thermal mass correction by $S$ and this dominates over the first term as long as $\tilde{\lambda}_{S \phi}>12 g_{B-L}^{2}(T / \tilde{T})^{2}$. Then, the end of the thermal inflation is determined by the last two terms of Eq. (93) with $\langle h\rangle=v_{\mathrm{QCD}}{ }^{2}$;

$$
\tilde{T}_{\text {end }}=\sqrt{\frac{24}{\tilde{\lambda}_{S \phi}}} \times g_{B-L}\left(\frac{m_{H}}{m_{Z^{\prime}}}\right) v_{\mathrm{QCD}}
$$

As a result, the dilution factor during the thermal inflation is given by

$e^{-4 \Delta N}=\left(\frac{\tilde{T}_{\text {end }}}{\tilde{T}_{T I}}\right)^{4}=\frac{30 g_{B-L}^{4}}{\pi^{2} N}\left(\frac{24}{\tilde{\lambda}_{S \phi}}\right)^{2}\left(\frac{m_{H}}{m_{Z^{\prime}}}\right)^{4} \frac{v_{\mathrm{QCD}}^{4}}{V_{\mathrm{TI}}}$,

where we have used the relation (75) at $\tilde{T}=\tilde{T}_{\mathrm{TI}}$. For given values of $g_{B-L}$ and $\tilde{\lambda}_{S \phi}$, the above dilution factor becomes tiny if $m_{Z^{\prime}}$ is heavy. On the other hand, as we will see below, $m_{Z^{\prime}}$ must be typically lighter than $10 \mathrm{TeV}$ for sufficient dilution of axions.

\section{Dilution of axions}

We now estimate $r_{A}$ in the massless case. As well as the massive case, the oscillating temperature is determined by $m_{A 0}=3 H$

\footnotetext{
${ }^{2}$ As discussed below, $m_{Z^{\prime}} \sim 10 \mathrm{TeV}$ is allowed in the massless case because we can easily earn the dilution of $S$ compared to the massive case. For such a small value of $m_{Z^{\prime}}$, the third term in Eq. (93) is already bigger than the first term due to the largeness of the Higgs vev $\langle h\rangle \sim 100 \mathrm{GeV}$ at $T=T_{\mathrm{QCD}}$.
} 


$$
\begin{aligned}
\tilde{T}_{\mathrm{osc}} & =\left(\frac{10}{\pi^{2} N}\right)^{1 / 4}\left(m_{A 0} M_{p l}\right)^{1 / 2} \\
& =2.2 \times 10^{-3} \mathrm{GeV}\left(\frac{10^{19}}{N}\right)^{1 / 4}\left(\frac{m_{A 0}}{6 \times 10^{-6} \mathrm{eV}}\right)^{1 / 2},
\end{aligned}
$$

which is much smaller than $\tilde{T}_{\mathrm{TI}}$. Thus, the axion field does not evolve until the beginning of thermal inflation and we can again use the same result of $r_{A}$,

$r_{A} \sim\left(\frac{2 \tan \left(\bar{\theta}_{\mathrm{ini}} / 2\right)}{0.3}\right)^{2}\left(\frac{f_{A}}{10^{12} \mathrm{GeV}}\right)^{1.16} \exp (-2 \eta \times \Delta N)$, where $\eta$ is the same as Eq. (71) and

$$
\Delta N=\log \left(\tilde{T}_{\mathrm{TI}} / \tilde{T}_{\text {end }}\right) .
$$

From the observational constraint for $R \sim 0.01$ in Fig. 2 and $r_{A}$ in Eq. (15), $r_{A}$ must be as tiny as $10^{-4}$. We will thus plot the excluded region of $r_{A}<8.2 \times 10^{-3} R$ in the $\tilde{\lambda}_{S H^{-}} m_{Z}^{\prime}$ plane for a given value of $f_{A}$.

\section{Dilution of initial abundance of $S$}

Let us now evaluate the relic abundance of $S$ in the massless case. Instead of Eq. (73), the relic abundance of massless components of $S$ is estimated as

$$
\begin{aligned}
\left.\frac{\rho_{S}}{\rho_{\gamma}}\right|_{\text {today }} & =\left.\frac{\rho_{S}}{\rho_{\gamma}}\right|_{T=T_{R}}=\left(\frac{g_{R} \pi^{2}}{30} \frac{T_{R}^{4}}{V_{\mathrm{TI}}}\right)^{4 / 3} \times e^{-4 \Delta N} \times \frac{V_{\mathrm{TI}}}{\left.\rho_{\gamma}\right|_{T=T_{R}}} \\
& =\frac{N-1}{2}\left(\frac{g_{R} \pi^{2}}{30} \frac{T_{R}^{4}}{V_{\mathrm{TI}}}\right)^{4 / 3}\left(\frac{\tilde{T}_{\text {end }}}{T_{R}}\right)^{4} \\
& \sim \frac{3.4 \times 10^{-7}}{\tilde{\lambda}_{S \phi}^{2}}\left(\frac{N}{10^{19}}\right)\left(\frac{g_{R}}{100}\right)^{4 / 3}\left(\frac{g_{B-L}}{0.1}\right)^{4}\left(\frac{v_{\mathrm{QCD}}}{150 \mathrm{MeV}}\right)^{4}\left(\frac{10 \mathrm{TeV}}{m_{Z^{\prime}}}\right)^{28 / 3}\left(\frac{T_{R}}{m_{H}}\right)^{4 / 3} .
\end{aligned}
$$

The above relic abundance contributes to the present energy density as dark radiation. Extra contribution to the number of relativistic species is defined by

$$
\rho_{\text {rad }}:=N_{\text {eff }} \frac{7}{8}\left(\frac{4}{11}\right)^{4 / 3} \rho_{\gamma} .
$$

The current bound by Planck 2018 [1] is

$$
N_{\text {eff }}=2.99_{-0.33}^{+0.34} \quad(95 \% \mathrm{CL}) .
$$

In the standard cosmology, $N_{\text {eff }}=3.046$ by neutrinos. Thus, Eq. (99) corresponds to

$$
\Delta N_{\mathrm{eff}}=\frac{8}{7}\left(\frac{11}{4}\right)^{4 / 3} \rho_{S} /\left.\rho_{\gamma}\right|_{\text {today }}=1.5 \times 10^{-6} \times \cdots
$$

On the other hand, the massive component of the $O(N)$ scalars becomes nonrelativistic when the temperature $T$ is of the same order as $m_{S}=\left(\tilde{\lambda}_{S H} / N\right)^{1 / 2} v$. With $\left.\rho_{S}\right|_{\text {today }}$ being the energy density of the $N-1$ massless components, the energy density of the massive component is evaluated as

$$
\begin{aligned}
r_{S} & =\frac{\rho_{S}^{\text {Massive }}}{\rho_{\mathrm{DM}}}=\left.\left.\frac{1}{N-1} \frac{\rho_{S}}{\rho_{\gamma}}\right|_{\text {today }}\left(\rho_{\gamma} / \rho_{\mathrm{DM}}\right)\right|_{\text {today }} \frac{m_{S}}{T_{0}} \\
& \sim 10^{-20} \times \frac{\tilde{\lambda}_{S H}^{1 / 2}}{\tilde{\lambda}_{S \phi}^{2}}\left(\frac{10^{19}}{N}\right)^{1 / 2}\left(\frac{g_{R}}{100}\right)^{4 / 3}\left(\frac{g_{B-L}}{0.01}\right)^{4}\left(\frac{v_{\mathrm{QCD}}}{150 \mathrm{MeV}}\right)^{4}\left(\frac{10 \mathrm{TeV}}{m_{Z^{\prime}}}\right)^{28 / 3}\left(\frac{T_{R}}{m_{H}}\right)^{4 / 3}
\end{aligned}
$$

which is tiny and we can safely neglect the isocurvature of the $O(N)$ scalar.

\section{Productions of $S$ after thermal inflation}

We also have to consider the production of $S$ after the secondary reheating. Here we consider a case of low reheating temperature, $T_{R} \leq m_{H}$, and focus on $h h \rightarrow S S$.
In the following, we set $a=1$ at $T=T_{R}$ i.e., $T=T_{R} a^{-1}$. The Boltzmann equation is

$$
\dot{\rho}_{S}+4 H \rho_{S}=\frac{N \lambda_{S H}^{2}}{16 \pi m_{H}} n_{H}^{2}=\frac{\tilde{\lambda}_{S H}^{2}}{16 \pi N m_{H}}\left(\frac{m_{H} T}{2 \pi}\right)^{3} e^{-2 m_{H} / T},
$$



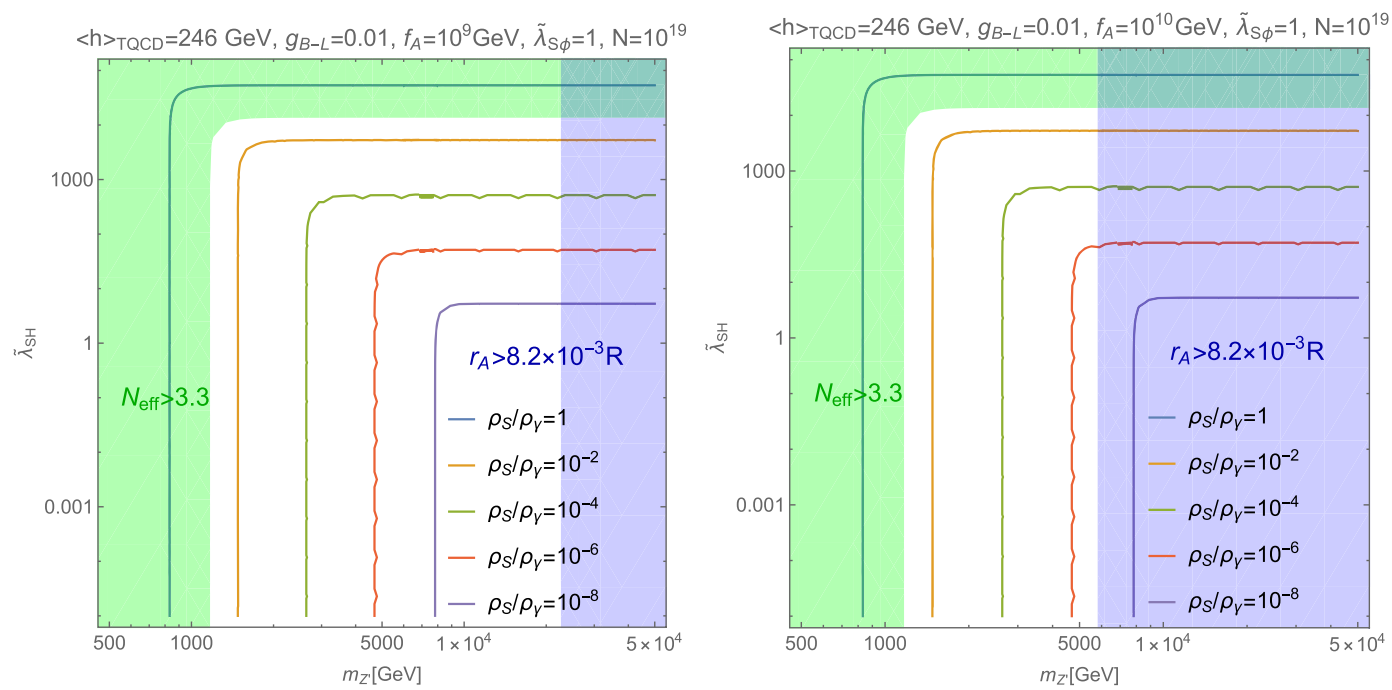

FIG. 6. Allowed parameter regions in the case of massless $S$. The left (right) panel corresponds to $f_{A}=10^{9}\left(10^{10}\right) \mathrm{GeV}$.

from which the ratio $Y:=\rho_{S} / \rho_{\mathrm{SM}}$ obeys

$$
\begin{aligned}
\frac{d Y}{d a} & =\frac{\tilde{\lambda}_{S H}^{2}}{16 \pi N m_{H}}\left(\frac{m_{H} T}{2 \pi}\right)^{3} \frac{e^{-2 m_{H} / T}}{a H \rho_{\mathrm{SM}}} \\
& =d\left(m_{H} / T_{R}\right)\left(m_{H} a / T_{R}\right)^{2} e^{-2\left(m_{H} / T_{R}\right) a},
\end{aligned}
$$

where we defined

$$
\begin{aligned}
d & :=\frac{3^{1 / 2} \tilde{\lambda}_{S H}^{2}}{2^{7} \pi^{4} N}\left(\frac{30}{\pi^{2} g_{\mathrm{SM}}}\right)^{3 / 2} \frac{M_{p l}}{m_{H}} \\
& =1.4 \times 10^{-9} \times \tilde{\lambda}_{S H}^{2}\left(\frac{100}{g_{\mathrm{SM}}}\right)^{3 / 2}\left(\frac{10^{19}}{N}\right) .
\end{aligned}
$$

The integration of Eq. (105) gives

$$
Y \sim d / 4 \text { for } a \gg T_{R} / m_{H},
$$

which contributes to the dark radiation as well as the initial abundance of $S$. Thus, if $N$ is sufficiently large with $\tilde{\lambda}_{S H}$ fixed, we can safely avoid the overproduction of $S$. As for the massive component, it is easy to see that its production is also negligible by the same calculation as Eq. (103). In D, we also discuss the productions via $f \bar{f} \rightarrow S S$, which is found to be subdominant compared to $h h \rightarrow S S$.

In Fig. 6, we show the allowed parameter region on the $\left(\tilde{\lambda}_{S H}, m_{Z^{\prime}}\right)$ plane. The left (right) panel corresponds to $f_{A}=10^{9}\left(10^{10}\right) \mathrm{GeV}$. In the figure, the green regions are excluded by the constraint on the dark radiation Eq. (101) while the blue regions are excluded by the isocurvature constraint of the CMB observations, $r_{A}<8.2 \times 10^{-3} R$. As a result, one can see that $m_{Z^{\prime}}$ is constrained to be $1 \mathrm{TeV} \lesssim$ $m_{Z^{\prime}} \lesssim 22 \mathrm{TeV}(6 \mathrm{TeV})$ when $f_{A}=10^{9}\left(10^{10}\right) \mathrm{GeV}$.

\section{CONCLUSIONS}

In the paper, we have investigated a possibility of the axion-CMB scenario in particle physics models with classical conformality $(C C)$. In such CC models, the early universe often experiences supercooled era of the EW symmetry breaking until the temperature of the universe cools down to QCD scale $T_{\mathrm{QCD}}$, and the thermal inflation naturally occurs at low temperature. On the other hand, the axion-CMB scenario in which the CMB fluctuations are assumed to be generated from the primordial axion fluctuations requires that the axion abundance must be sufficiently diluted after the axion potential is generated at $T_{\mathrm{QCD}}$. Thus, if the thermal inflation in the $\mathrm{CC}$ models occurs below $T_{\mathrm{QCD}}$, CC models can be candidates for particle physics models realizing the axion-CMB scenario.

In this paper, we have particularly studied the CC $B-L$ model with an additional extension of $O(N)$ scalars $S$. Such an extension is necessary in order to generate sufficiently large amplitude of axion potential at QCD temperature. Namely, the Higgs vev in the supercooled era of EW symmetry must be generated by a negative scalar coupling with $S$ and become as large as $10^{2} \mathrm{GeV}$. This requirement is fulfilled by considering decoupled evolutions of the SM sector and $O(N)$ sector where the temperature of each sector is completely different.

We have investigated various observational constraints of CMB observations and the present abundance of axions and the additional particles $S$, and shown that, if $S$ is massless, there is a parameter region in which all the constraints are satisfied.

\section{ACKNOWLEDGMENTS}

The work of S. I. is supported in part by the Grant-in-Aid for Scientific research, No. 18H03708, No. 16H06490. 


\section{APPENDIX A: BOLTZMANN EQUATIONS}

In this Appendix, we summarize the basic calculations of Boltzmann equations [52,53].

\section{Thermal average of cross sections}

The thermal average of cross section by the Maxwell-Boltzmann distribution is given by [52]

$$
\langle\sigma v\rangle=\frac{1}{T^{2} x K_{2}(x)^{2}} \int_{1}^{\infty} d y\left(4 m^{2} \sigma\right)(y-1) \sqrt{y} K_{1}(2 x \sqrt{y}), \quad x=m / T, \quad y=s /\left(4 m^{2}\right),
$$

where

$$
K_{\nu}(z)=\frac{\pi^{1 / 2}(z / 2)^{\nu}}{\Gamma(\nu+1 / 2)} \int_{1}^{\infty} d t e^{-z t}\left(t^{2}-1\right)^{\nu-1 / 2}
$$

is the modified bessel function of the second kind. The cross sections of $S S \leftrightarrow H H$ are

$$
\sigma_{S S \rightarrow H H}(s)=\frac{\lambda_{S H}^{2}}{16 \pi s} \sqrt{\frac{s-4 m_{H}^{2}}{s-4 m_{S}^{2}}}, \quad \sigma_{H H \rightarrow S S}(s)=\frac{\lambda_{S H}^{2}}{16 \pi s} \sqrt{\frac{s-4 m_{S}^{2}}{s-4 m_{H}^{2}}},
$$

from which we obtain

$$
\begin{gathered}
\left\langle\sigma_{S S \rightarrow H H} v\right\rangle=\frac{\lambda_{S H}^{2}}{16 \pi \tilde{T}^{2} x_{S} K_{2}\left(x_{S}\right)^{2}} \int_{\left(m_{H} / m_{S}\right)^{2}}^{\infty} d y y^{-1 / 2} \sqrt{(y-1)\left(y-\left(m_{H} / m_{S}\right)^{2}\right)} K_{1}\left(2 x_{S} \sqrt{y}\right), \\
\left\langle\sigma_{H H \rightarrow S S} v\right\rangle=\frac{\lambda_{S H}^{2}}{16 \pi T^{2} x_{H} K_{2}\left(x_{H}\right)^{2}} \int_{\left(m_{S} / m_{H}\right)^{2}}^{\infty} d y y^{-1 / 2} \sqrt{(y-1)\left(y-\left(m_{S} / m_{h}\right)^{2}\right)} K_{1}\left(2 x_{H} \sqrt{y}\right),
\end{gathered}
$$

where $x_{S}=m_{S} / \tilde{T}, x_{H}=m_{H} / T$. Our main focus is Eq. (A4) because we assume that $S$ is the dominant component during the period between the primordial preheating and the thermal inflation. In the massive $S$ case, we saw that $m_{H} / m_{S}$ is always negligible as shown in Eq. (47). Thus, we can neglect the higher order terms of $\left(m_{H} / m_{S}\right)^{2}$ in the rhs in Eq. (A4). Within this approximation, the integration becomes

$$
\int_{1}^{\infty} d y(y-1)^{1 / 2} K_{1}\left(2 x_{S} \sqrt{y}\right)=K_{1}\left(x_{S}\right)^{2} / 2 x_{S},
$$

which leads to

$$
\left\langle\sigma_{S S \rightarrow H H} v\right\rangle=\frac{\lambda_{S H}^{2}}{32 \pi \tilde{T}^{2} x_{S}^{2}}\left(\frac{K_{1}\left(x_{S}\right)}{K_{2}\left(x_{S}\right)}\right)^{2} .
$$

When $T \gg m$, i.e., $x \ll 1$, the bessel functions are expanded as

$$
\left(\frac{K_{1}(x)}{K_{2}(x)}\right)^{2}=\frac{x^{2}}{4}+\mathcal{O}\left(x^{3}\right)
$$

from which we have

$$
\left\langle\sigma_{S S \rightarrow H H} v\right\rangle \sim \frac{\lambda_{S H}^{2}}{128 \pi \tilde{T}^{2}} \quad \text { for } \tilde{T} \gg m_{S}
$$

On the other hand, in the non-relativistic limit $\tilde{T} \ll m_{S}$, i.e., $x_{S} \ll 1$, the bessel functions are expanded as

$$
\left(\frac{K_{1}(x)}{K_{2}(x)}\right)^{2}=1-\frac{3}{x}+\mathcal{O}\left(x^{-2}\right),
$$

from which we have

$$
\left\langle\sigma_{S S \rightarrow H H} v\right\rangle \sim \frac{\lambda_{S H}^{2}}{16 \pi m_{S}^{2}}\left(1-\frac{3 \tilde{T}}{m_{S}}\right) \quad \text { for } \tilde{T} \ll m_{S} .
$$

\section{Boltzmann equations}

In general, the Boltzmann equation for particle species 1 is given by

$$
\frac{\partial n_{1}}{\partial t}+3 H n_{1}=\int \frac{d^{3} \mathbf{p}}{(2 \pi)^{3} E(p)} \hat{C}\left[f_{1}\right]
$$

where the collision term is 


$$
\begin{aligned}
\int C\left[f_{1}\right] \frac{d^{3} p}{(2 \pi)^{3}}= & -\sum_{\text {dof }} \int \frac{d^{3} p_{1}}{(2 \pi)^{3} 2 E_{1}} \frac{d^{3} p_{2}}{(2 \pi)^{3} 2 E_{2}} \frac{d^{3} p_{3}}{(2 \pi)^{3} 2 E_{3}} \frac{d^{3} p_{4}}{(2 \pi)^{3} 2 E_{4}} \\
& \times\left\{f_{1} f_{2}\left(1 \pm f_{3}\right)\left(1 \pm f_{4}\right)\left|\mathcal{M}_{12 \rightarrow 34}\right|^{2}-f_{3} f_{4}\left(1 \pm f_{1}\right)\left(1 \pm f_{2}\right)\left|\mathcal{M}_{34 \rightarrow 12}\right|^{2}\right\} \\
& \times(2 \pi)^{4} \delta\left(p_{1}+p_{2}-p_{3}-p_{4}\right) .
\end{aligned}
$$

In our model, 1,2 corresponds to $S_{i}(i=1,2, \ldots, N)$, and 3,4 corresponds to the SM Higgs, and vice versa. When the system is not experiencing BEC and Fermi degeneracy, it is good to approximate $\left(1 \pm f_{l}\right)$ by 1 . Then, assuming thermal (kinetic) equilibrium in each particles, we have

$$
\dot{n}_{i}+3 H n_{i}=-\left\langle\sigma_{S S \rightarrow H H} v\right\rangle n_{i}^{2}+\left\langle\sigma_{H H \rightarrow S S} v\right\rangle n_{H}^{2}
$$

where $\left\langle\sigma_{S S(H H) \rightarrow H H(S S)} v\right\rangle$ is the thermal average Eqs. (A4) (A5).

\section{APPENDIX B: REHEATING TEMPERATURE AFTER THERMAL INFLATION}

In this Appendix, we give a rough estimation of the reheating temperature $T_{R}$ after the thermal inflation. It is defined as the temperature of the SM radiation at the moment when the coherent oscillation of the flaton field $\phi$ is disintegrated into radiation. We assume that the reheating is completed either by $\phi$ 's decay into two SM Higgs particles or by $\phi$ 's scattering with the Higgs in the SM thermal bath. Reheating by $\phi$ decay into SM particles via the scalar mixing is neglected for simplicity.

We restrict ourselves to the case where the $B-L$ gauge boson $Z^{\prime}$ is much heavier than the right-handed neutrino $N$ and the coefficient in Eq. (17) is dominantly given by $Z^{\prime}$. Then, the mass of $\phi$ field at the true vacuum is obtained as $m_{\phi} \sim g_{B-L} m_{Z^{\prime}}$. If $m_{\phi} \gtrsim m_{h}$, or equivalently,

$$
g_{B-L} \gtrsim m_{h} / m_{Z^{\prime}},
$$

$\phi$ can decay into two Higgs particles with the rate

$$
\Gamma_{\mathrm{d}} \sim \frac{\left(\lambda_{\phi H} v_{\phi}\right)^{2}}{m_{\phi}} \sim g_{B-L} \frac{m_{h}^{4}}{m_{Z^{\prime}}^{3}} .
$$

Equating this with the Hubble expansion rate $H \sim T^{2} / M_{p l}$, we get

$$
T_{R}^{(\mathrm{d} 1)}=\left(g_{B-L} m_{Z^{\prime}} M_{p l}\right)^{1 / 2}\left(m_{h} / m_{Z^{\prime}}\right)^{2} .
$$

The derivation is valid as far as the thermal mass of the Higgs is below $m_{\phi}$. The condition $m_{h}\left(T_{R}^{(d 1)}\right) \sim T_{R}^{(\mathrm{d} 1)} \lesssim m_{\phi}$ is rewritten as

$$
g_{B-L} \lesssim x:=\frac{m_{h}^{4} M_{p l}}{m_{Z^{\prime}}^{5}} .
$$

If the condition is not satisfied, the decay process is kinematically forbidden until the temperature is reduced to $T=m_{\phi} \sim g_{B-L} m_{Z^{\prime}}$. In this case, the reheating temperature is given by $T_{R}^{(\mathrm{d} 2)}=m_{\phi} \sim g_{B-L} m_{Z^{\prime}}$. To summarize, the reheating temperature by decay is

$$
T_{R}^{(\mathrm{d})} \sim \min \left\{T_{R}^{(\mathrm{d} 1)}, m_{\phi}\right\} \leq m_{\phi} .
$$

Note that the scalar field $\phi$ acquires mass through the Coleman Weinberg mechanism, and $m_{\phi}$ is generally small. In the $\mathrm{CC} B-L$ model, in particular, it cannot be much heavier than the SM Higgs; $m_{\phi} \leq m_{H}$. Thus the reheating temperature by the decay process is also expected to be not so high compared to $m_{H}$.

On the other hand, the scattering process is not thermally blocked. Once the SM thermal bath is generated, the coherent oscillation is disintegrated into $\phi$ particles due to scattering with the Higgs in the bath. The interaction rate in the relativistic region $T \gtrsim m_{\phi}$ is given by

$$
\Gamma_{\mathrm{s}} \sim \lambda_{\phi H}^{2} T \sim g_{B-L}^{4}\left(\frac{m_{h}}{m_{Z^{\prime}}}\right)^{4} T,
$$

whereas $\Gamma_{\mathrm{s}} \sim \lambda_{\phi H}^{2} T^{3} / m_{\phi}$ for $T \lesssim m_{\phi}$. By comparing it with the Hubble, the reheating temperature by the scattering process is given by ${ }^{3}$

$$
T_{R}^{(\mathrm{s})} \sim g_{B-L}^{4} M_{p l}\left(\frac{m_{h}}{m_{Z^{\prime}}}\right)^{4}
$$

as far as $T_{R}^{(\mathrm{s})} \gtrsim m_{\phi}$ is satisfied, i.e.,

$$
g_{B-L}^{3} \gtrsim \frac{m_{Z^{\prime}}^{5}}{m_{h}^{4} M_{p l}}=1 / x .
$$

If it is not satisfied, we have $T_{R}^{(\mathrm{s})}=0$. For example, if $m_{Z^{\prime}}=10 \mathrm{TeV}$ and $g_{B-L}=0.01, T_{R}^{(s)} \sim 600 \mathrm{GeV}$.

\footnotetext{
${ }^{3}$ The $\phi$ particles produced in the scattering process have yet to decay or pair-annihilate into the SM particles. Therefore, this $T_{R}^{(\mathrm{s})}$ should be regarded as a maximum value of the reheating temperature.
} 
The reheating temperature is given by $T_{R}=$ $\max \left\{T_{R}^{(\mathrm{d})}, T_{R}^{(\mathrm{s})}\right\}$. Comparing Eq. (B5) and Eq. (B7), we have $T_{R}=T_{R}^{(\mathrm{s})}$ when the condition (B8) is satisfied. Provided $g_{B-L}<1$, when the condition (B8) is violated, the inequality $x>1 / g_{\mathrm{B}-\mathrm{L}}^{3}>g_{\mathrm{B}-\mathrm{L}}$ follows and Eq. (B4) is always satisfied. Thus as far as Eq. (B1) is satisfied,

$$
T_{R} \sim T_{R}^{(\mathrm{d} 1)}<m_{\phi} \text { for } g_{B-L} \gtrsim \frac{m_{h}}{m_{Z^{\prime}}} .
$$

If Eq. (B1) is not satisfied, the decay process is forbidden, and the reheating is only possible because of the scattering process. Then, the condition (B8) must be satisfied so that $T_{R}=T_{R}^{(\mathrm{s})}$ is non-zero.

\section{APPENDIX C: $S$ PRODUCTION DURING REHEATING}

In the body of the paper, we have considered the production of the $O(N)$ scalar particles after the reheating is discussed. Here, we will consider the production during the reheating of thermal inflation and show that it does not surpass the production after the reheating.

Neglecting the Boltzmann suppression, the Boltzmann equation for the energy density is given as

$$
\dot{\rho}_{S}+4 H \rho_{S} \sim N \lambda_{S H}^{2} T \rho_{\mathrm{SM}},
$$

where $\rho_{\mathrm{SM}} \sim T^{4}$ and $H \simeq \sqrt{\rho_{\phi} / 3 M_{p l}^{2}}=H_{R} a^{-3 / 2}$ with $H_{R}=\left.\sqrt{\rho_{\phi} / 3 M_{p l}^{2}}\right|_{T=T_{R}} \cdot \rho_{\mathrm{SM}}$ first grows by its production from coherent oscillation of $\phi$, and then it is expected to decrease with a scaling coefficient $x$ as $T=T_{R} a^{-x}$. After the reheating, the coefficient approaches $x=1$. Since the energy density of coherent oscillation behaves as $a^{-3}$ and $\rho_{\phi} \sim \rho_{\mathrm{SM}}$ at $T_{R}, x$ is expected to be $x<3 / 4$ so that $\rho_{\mathrm{SM}}<$ $\rho_{\phi}$ before $T_{R}$.

Then, the Boltzmann equation becomes

$$
\frac{d\left(\rho_{S} a^{4}\right)}{d a} \sim \frac{\tilde{\lambda}_{S H}^{2}}{N} \frac{T_{R}^{5}}{H_{R}} a^{\frac{9}{2}-5 x}
$$

and is solved as

$\rho_{S} \sim \rho_{S \text {, end }}\left(\frac{a_{\text {end }}}{a}\right)^{4}+\frac{d T_{R}^{4} a^{-4}}{11 / 2-5 x}\left(a^{\frac{11}{2}-5 x}-a_{\text {end }}^{\frac{11}{2}-5 x}\right)$

with $d$ defined in Eq. (106). Since $\frac{11}{2}-5 x>0$, when the reheating process ends at $a=1$, the energy density of produced $S$ is given by

$$
\left.\rho_{S}\right|_{T=T_{R}} \sim d T_{R}^{4}
$$

because of $a_{\text {end }} \ll 1$. Thus the $S$ particles produced early in the reheating period are diluted and the ones produced around $a=1$ is dominant, which justifies the analysis in the body of the paper.

\section{APPENDIX D: $f \bar{f} \rightarrow S S$}

In this Appendix, we consider production of $S$ after the secondary reheating via the process $f \bar{f} \rightarrow S S$ where $f$ is a $\mathrm{SM}$ fermion. The cross section is

$\sigma_{f \bar{f} \rightarrow S S}(s)=\frac{y_{f}^{2} v^{2} \lambda_{S H}^{2}}{64 \pi} \frac{1}{\left(s-m_{H}^{2}\right)^{2}+m_{H}^{2} \Gamma_{H}^{2}} \frac{s-4 m_{f}^{2}}{s}$,

where $\Gamma_{H}=4 \mathrm{MeV}$ is the total decay width of the SM Higgs. The thermal average is now given by

$$
\begin{aligned}
\left\langle\sigma_{f \bar{f} \rightarrow S S} v\right\rangle= & \frac{v^{2} \lambda_{S H}^{2}}{64 \pi T^{2} x K_{2}(x)^{2} \times 4 m_{f}^{2}} \\
& \times \int_{1}^{\infty} d y \frac{(y-1)^{2} y^{-1 / 2}}{\left(y-m_{H}^{2} /\left(4 m_{f}^{2}\right)\right)^{2}+m_{H}^{2} \Gamma^{2} /\left(4 m_{f}^{2}\right)^{2}} \\
& \times K_{1}(2 x \sqrt{y}), \quad \text { (D2) } \\
& x=m_{f} / T, \quad y=s /\left(4 m_{f}^{2}\right) . \quad \text { (D3) }
\end{aligned}
$$

The Boltzmann equation is

$\dot{\rho}_{S}+4 H \rho_{S}=N T\left\langle\sigma_{f \bar{f} \rightarrow S S} v\right\rangle n_{f}(T)^{2},\left.\quad \rho_{S}\right|_{T=T_{R}}=0$,

where

$n_{f}(T)=\int \frac{d 3 p}{(2 \pi)^{3}} \frac{1}{e^{\beta E(p)}+1}, \quad E(p)=\sqrt{p^{2}+m_{f}^{2}}$.

In Fig. 7, we show the numerical calculations of Eq. (D4) where the different colors correspond to the different values of $\tilde{\lambda}_{S H}$.

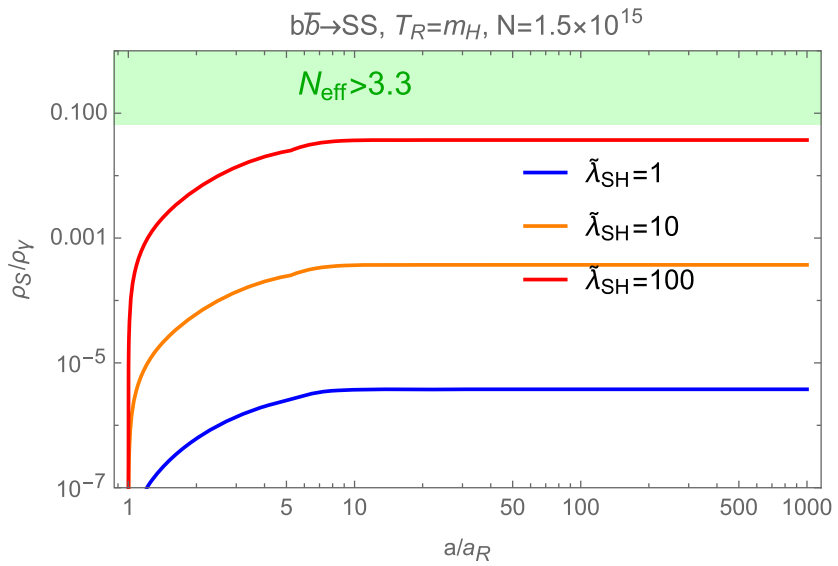

FIG. 7. Production of $S$ via $b \bar{b}$ after the reheating of thermal inflation. 
[1] N. Aghanim et al. (Planck Collaboration), Planck 2018 results. VI. Cosmological parameters, Astron. Astrophys. 641, A6 (2020).

[2] Y. Akrami et al. (Planck Collaboration), Planck 2018 results. X. Constraints on inflation, Astron. Astrophys. 641, A10 (2020).

[3] Y. Akrami et al. (Planck Collaboration), Planck 2018 results. IX. Constraints on primordial non-Gaussianity, Astron. Astrophys. 641, A9 (2020).

[4] D. H. Lyth and D. Wands, Generating the curvature perturbation without an inflaton, Phys. Lett. B 524, 5 (2002).

[5] K. Enqvist and M. S. Sloth, Adiabatic CMB perturbations in pre-big bang string cosmology, Nucl. Phys. B626, 395 (2002).

[6] T. Moroi and T. Takahashi, Effects of cosmological moduli fields on cosmic microwave background, Phys. Lett. B 522, 215 (2001); Erratum, Phys. Lett. B 539, 303 (2002).

[7] D. H. Lyth, C. Ungarelli, and D. Wands, The primordial density perturbation in the curvaton scenario, Phys. Rev. D 67, 023503 (2003).

[8] C. Gordon and A. Lewis, Observational constraints on the curvaton model of inflation, Phys. Rev. D 67, 123513 (2003).

[9] K. Dimopoulos, D. Lyth, A. Notari, and A. Riotto, The curvaton as a pseudoNambu-Goldstone boson, J. High Energy Phys. 07 (2003) 053.

[10] M. Sasaki, J. Valiviita, and D. Wands, Non-Gaussianity of the primordial perturbation in the curvaton model, Phys. Rev. D 74, 103003 (2006).

[11] P. Chingangbam and Q.-G. Huang, The curvature perturbation in the axion-type curvaton model, J. Cosmol. Astropart. Phys. 04 (2009) 031.

[12] K. Enqvist, S. Nurmi, O. Taanila, and T. Takahashi, Nongaussian fingerprints of self-interacting curvaton, J. Cosmol. Astropart. Phys. 04 (2010) 009.

[13] K. Nakayama and J. Yokoyama, Gravitational wave background and non-Gaussianity as a probe of the curvaton scenario, J. Cosmol. Astropart. Phys. 01 (2010) 010.

[14] C. T. Byrnes, K. Enqvist, and T. Takahashi, Scaledependence of non-gaussianity in the curvaton model, J. Cosmol. Astropart. Phys. 09 (2010) 026.

[15] S. Iso, K. Kawana, and K. Shimada, QCD axions and CMB anisotropy, Phys. Rev. D 102, 103513 (2020).

[16] R. Peccei and H. R. Quinn, CP Conservation in the Presence of Instantons, Phys. Rev. Lett. 38, 1440 (1977).

[17] F. Wilczek, Problem of Strong $P$ and $T$ Invariance in the Presence of Instantons, Phys. Rev. Lett. 40, 279 (1978).

[18] S. Weinberg, A New Light Boson?, Phys. Rev. Lett. 40, 223 (1978).

[19] J. E. Kim, Weak Interaction Singlet and Strong CP Invariance, Phys. Rev. Lett. 43, 103 (1979).

[20] M. A. Shifman, A. Vainshtein, and V. I. Zakharov, Can confinement ensure natural $C P$ invariance of strong interactions?, Nucl. Phys. B166, 493 (1980).

[21] A. Zhitnitsky, On possible suppression of the axion hadron interactions. (In Russian), Sov. J. Nucl. Phys. 31, 260 (1980).

[22] M. Dine, W. Fischler, and M. Srednicki, A simple solution to the strong $C P$ problem with a harmless axion, Phys. Lett. 104B, 199 (1981).
[23] J. E. Kim and G. Carosi, Axions and the strong $C P$ problem, Rev. Mod. Phys. 82, 557 (2010); Erratum, Rev. Mod. Phys. 91, 049902 (2019).

[24] G. Grilli di Cortona, E. Hardy, J. Pardo Vega, and G. Villadoro, The QCD axion, precisely, J. High Energy Phys. 01 (2016) 034.

[25] D. H. Lyth and E. D. Stewart, Thermal inflation and the moduli problem, Phys. Rev. D 53, 1784 (1996).

[26] J.-O. Gong, N. Kitajima, and T. Terada, Curvaton as dark matter with secondary inflation, J. Cosmol. Astropart. Phys. 03 (2017) 053.

[27] T. Hambye, A. Strumia, and D. Teresi, Super-cool dark matter, J. High Energy Phys. 08 (2018) 188.

[28] P. Baratella, A. Pomarol, and F. Rompineve, The supercooled Universe, J. High Energy Phys. 03 (2019) 100.

[29] S. Iso, N. Okada, and Y. Orikasa, Classically conformal $B^{-}$ L extended standard model, Phys. Lett. B 676, 81 (2009).

[30] S. Iso, N. Okada, and Y. Orikasa, The minimal B-L model naturally realized at TeV scale, Phys. Rev. D 80, 115007 (2009).

[31] S. Iso and Y. Orikasa, TeV Scale B-L model with a flat Higgs potential at the Planck scale: In view of the hierarchy problem, Prog. Theor. Exp. Phys. 2013, 023 B08 (2013).

[32] S. Iso, P. D. Serpico, and K. Shimada, QCD-Electroweak First-Order Phase Transition in a Supercooled Universe, Phys. Rev. Lett. 119, 141301 (2017).

[33] Y. Hamada, H. Kawai, K.-y. Oda, and K. Yagyu, Dark matter in minimal dimensional transmutation with multicritical-point principle, J. High Energy Phys. 01 (2021) 087.

[34] Y. Hamada, H. Kawai, K. Kawana, K.-y. Oda, and K. Yagyu, Minimal scenario of criticality for electroweak scale, neutrino masses, dark matter, and inflation, arXiv:2102 .04617 .

[35] S. Weinberg, Gauge and global symmetries at high temperature, Phys. Rev. D 9, 3357 (1974).

[36] P. Meade and H. Ramani, Unrestored Electroweak Symmetry, Phys. Rev. Lett. 122, 041802 (2019).

[37] I. Baldes and G. Servant, High scale electroweak phase transition: Baryogenesis and symmetry non-restoration, J. High Energy Phys. 10 (2018) 053.

[38] A. Glioti, R. Rattazzi, and L. Vecchi, Electroweak baryogenesis above the electroweak scale, J. High Energy Phys. 04 (2019) 027.

[39] R. T. D'Agnolo and M. Low, Disorder and mimesis at hadron colliders, J. High Energy Phys. 08 (2019) 163.

[40] L. Di Luzio, M. Redi, A. Strumia, and D. Teresi, Coset cosmology, J. High Energy Phys. 06 (2019) 110.

[41] O. Matsedonskyi and G. Servant, High-temperature electroweak symmetry non-restoration from new fermions and implications for baryogenesis, J. High Energy Phys. 09 (2020) 012.

[42] S. Chaudhuri, C. Choi, and E. Rabinovici, Thermal order in large N conformal gauge theories, J. High Energy Phys. 04 (2021) 203.

[43] M. P. Hertzberg, M. Tegmark, and F. Wilczek, Axion cosmology and the energy scale of inflation, Phys. Rev. D 78, 083507 (2008).

[44] J. Preskill, M. B. Wise, and F. Wilczek, Cosmology of the invisible axion, Phys. Lett. 120B, 127 (1983). 
[45] L. Abbott and P. Sikivie, A cosmological bound on the invisible axion, Phys. Lett. B 120, 133 (1983).

[46] M. Dine and W. Fischler, The not so harmless axion, Phys. Lett. 120B, 137 (1983).

[47] M. Beltran, J. Garcia-Bellido, and J. Lesgourgues, Isocurvature bounds on axions revisited, Phys. Rev. D 75, 103507 (2007).

[48] O. Wantz and E. Shellard, Axion cosmology revisited, Phys. Rev. D 82, 123508 (2010).

[49] C. Hikage, M. Kawasaki, T. Sekiguchi, and T. Takahashi, CMB constraint on non-Gaussianity in isocurvature perturbations, J. Cosmol. Astropart. Phys. 07 (2013) 007.
[50] M. Kawasaki and K. Nakayama, Axions: Theory and cosmological role, Annu. Rev. Nucl. Part. Sci. 63, 69 (2013).

[51] E. Witten, Cosmological consequences of a light Higgs boson, Nucl. Phys. B177, 477 (1981).

[52] P. Gondolo and G. Gelmini, Cosmic abundances of stable particles: Improved analysis, Nucl. Phys. B360, 145 (1991).

[53] K. Ala-Mattinen and K. Kainulainen, Precision calculations of dark matter relic abundance, J. Cosmol. Astropart. Phys. 09 (2020) 040. 